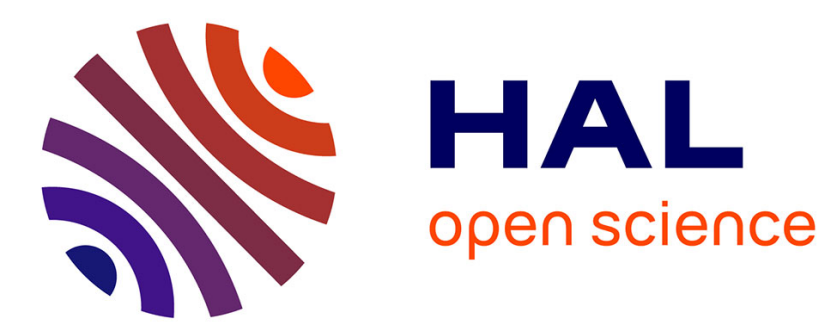

\title{
Fuzzy adaptive controller for MIMO nonlinear systems with known and unknown control direction
}

\author{
Abdesselem Boulkroune, Mohamed Tadjine, Mohammed M'Saad, Mondher
}

Farza

\section{- To cite this version:}

Abdesselem Boulkroune, Mohamed Tadjine, Mohammed M'Saad, Mondher Farza. Fuzzy adaptive controller for MIMO nonlinear systems with known and unknown control direction. Fuzzy Sets and Systems, 2009, pp.797-820. 10.1016/j.fss.2009.04.011 . hal-01059896

\section{HAL Id: hal-01059896 https://hal.science/hal-01059896}

Submitted on 5 Sep 2014

HAL is a multi-disciplinary open access archive for the deposit and dissemination of scientific research documents, whether they are published or not. The documents may come from teaching and research institutions in France or abroad, or from public or private research centers.
L'archive ouverte pluridisciplinaire HAL, est destinée au dépôt et à la diffusion de documents scientifiques de niveau recherche, publiés ou non, émanant des établissements d'enseignement et de recherche français ou étrangers, des laboratoires publics ou privés. 


\title{
Fuzzy adaptive controller for MIMO nonlinear systems with known and unknown control direction
}

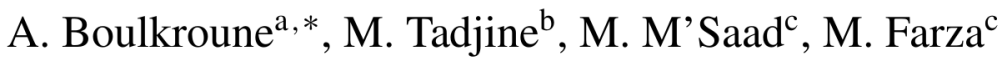 \\ ${ }^{a}$ Department of Automatic, University of Jijel, Jijel 18000, Algeria \\ ${ }^{\mathrm{b}}$ LCP, Department of the Electrical Engineering, ENP, El-Harrach, 16000, Algiers, Algeria \\ ${ }^{\mathrm{c}}$ GREYC, UMR 6072 CNRS, Université de Caen, ENSICAEN, 6 Bd Maréchal Juin, 14050 Caen Cedex, France
}

\begin{abstract}
This paper investigates fuzzy adaptive control schemes for a class of multi-input multi-output (MIMO) unknown nonlinear systems with known and unknown sign of the control gain matrix. Three fuzzy adaptive control schemes are developed. In the design of the second and third controller, we will exploit a decomposition of the control gain matrix into a symmetric positive-definite matrix, a diagonal matrix with diagonal entries +1 or -1 and a unity upper triangular matrix. The Nussbaum-type function is used to deal with the unknown control direction (i.e. the unknown sign of the control gain matrix). For updating the parameters of the fuzzy system, an adaptation proportional-integral (PI) law is proposed. Theoretical results are illustrated through two simulation examples.
\end{abstract}

Keywords: Fuzzy control; Adaptive control; Adaptation PI law; MIMO nonlinear systems; Nussbaum function

\section{Introduction}

In control engineering, most systems are multivariable in nature. It is certain that the control theory for multivariable systems will find immediate application in a wide variety of problems (space technology, electrical machines and robotics). The control of multivariable systems is a complicated problem due to the coupling that exists between the control inputs and the outputs. When multivariable systems are nonlinear and uncertain, their control problem becomes more challenging. In this area, theoretical results and constructive procedures for designing satisfactory controllers are really very few.

In the last decade, the nonlinear control based on the universal function approximators (fuzzy systems, neural networks) has received much attention [1,2,4,9,15,21,23,25-27,31]. Fuzzy control, in particular, has an impact in the control community because the fuzzy controllers provide a systematic and efficient framework to incorporate linguistic fuzzy information from human expert. Adaptive fuzzy control schemes for a class of multi-input multi-output (MIMO) nonlinear uncertain systems have been developed in $[3,5,8,11,13,14,16,19,28-30]$ thanks to the universal approximation theorem [31]. Indeed, this class has the following form: $y^{(r)}=F(x)+G(x) u$, where $x$ is the overall state vector, $u \in R^{p}$ the control input vector, $y \in R^{p}$ is the output vector, $F(x) \in R^{p}$ and $G(x)=\left[g_{i j}\right] \in R^{p \times p}$ are unknown continuous

\footnotetext{
* Corresponding author.

E-mail addresses: boulkroune2002@yahoo.fr (A. Boulkroune), tadjine@yahoo.fr (M. Tadjine), msaad@greyc.ensicaen.fr (M. M’Saad), mfarza@greyc.ensicaen.fr (M. Farza).
} 
nonlinear functions. The stability of the underlying closed-loop system is analyzed in Lyapunov sense. To cope with the fuzzy approximation error as well as external disturbances, these adaptive fuzzy controllers are commonly augmented by a robust control term that can be a supervisory control [8], sliding mode control [5,11,13,19,29,30], and/or $H^{\infty}$ control $[3,8,16,28]$. Conceptually, there exist two distinct approaches to design a fuzzy adaptive control system: direct and indirect approaches. In direct approach, $[3,14,16,19]$, the fuzzy system is used to estimate the so-called ideal control and the parameters of the fuzzy system are adjusted to meet the required control objective. Unlike the direct approach, the indirect adaptive approach uses fuzzy systems to estimate the system nonlinearities $F(x)$ and $G(x)$, and then a control law is calculated based on these estimates [3,5,8,11,13,16,19,28-30].

In fuzzy indirect adaptive control schemes, efforts have to be made to avoid the possible singularity problem when calculating the inverse of the estimated "decoupling matrix" (i.e. the estimate of the control gain matrix $G(x)$ ). In order to avoid the controller singularity, the authors of $[3,8,11,28,29]$ suggest to use a projection algorithm to keep the estimated fuzzy parameters of $\hat{G}\left(x, \theta_{g}\right)$ (where $\hat{G}\left(x, \theta_{g}\right)$ is the fuzzy estimate of the control gain matrix and $\theta_{g}$ represents the adjustable fuzzy parameters) inside a feasible set in which the singularity problem does not happen. Practically speaking, this solution has some disadvantages [31]. Indeed, it is usually requires a priori knowledge for the feasible parameter set and no systematic procedure is available for constructing such a set for general plant. In $[13,30]$, authors propose another alternative to avoid the controller singularity problem. It consists in the use of the regularized inverse of $\hat{G}\left(x, \theta_{g}\right)$ in the control law instead of its inverse.

A key assumption in these fuzzy adaptive control schemes $[3,5,8,11,13,14,16,19,28-30]$ is that the sign of the control gain matrix is known a priori. However, as stated in [32], the assumption on the sign knowledge of the so-called highfrequency gains (i.e. the sign of the control gain matrix) does not appear to be realistic in the general case. When there is no a priori knowledge about the sign of the control gains, the design of the adaptive controllers for MIMO nonlinear systems becomes more challenging. For a special class of MIMO nonlinear systems with unknown gain signs, adaptive (neural and fuzzy) control schemes have been, respectively, proposed in [33,34]. In these control schemes, the Nussbaum-type function [18] has been used to deal with the unknown control direction. Moreover, two restrictive modelling assumptions have been made to facilitate the stability analysis and the control design, namely: nonlinear systems with a lower triangular control structure and the boundedness of the high-frequency control gains.

In this paper, three fuzzy adaptive control schemes are developed for a class of MIMO uncertain nonlinear systems with known and unknown control direction.

The first fuzzy adaptive control scheme proposed, which is a direct scheme, is developed for a class of MIMO unknown nonlinear systems with symmetric control gain matrix whose sign is known. Bearing in mind the available results $[3,14,16,19]$, the main contributions of this work include:

(1) The assumptions on the control gain matrix are realistic and not too restrictive. Recall that in $[3,14,16,19]$, restrictive assumptions are made on the control gain matrix (see Remark 1 in Section 4.1).

(2) An adaptation proportional-integral (PI) law augmented by e-modification [17] is proposed to update the fuzzy parameters. Unlike $\sigma$-modification concept, the combination of e-modification concept with the robust term $K_{0} \operatorname{Sign}(S)$ in the proposed adaptive control scheme can assure a convergence of the tracking error to zero. As for the proportional term, it allows to fuzzy adaptive parameters a fast convergence. Indeed, the latter introduces a negative term in the Lyapunov's function derivative.

The second fuzzy adaptive control scheme proposed, being an indirect version, is designed for a class of MIMO unknown nonlinear systems with nonsymmetric or symmetric control gain matrix but with known signs of its leading principal minors. Compared with [3,5,8,11,13,16,19,28-30], there are four main contributions that are worth to be emphasized:

(1) Motivated by a matrix decomposition introduced in [6,7,12,35], we decompose $G(x)$ into the product of a symmetric positive-definite matrix, a diagonal matrix with +1 or -1 on the diagonal and a unity upper triangular matrix. It is worth noticing that the diagonal matrix elements are nothing than the ratios of the signs of the leading principal minors of the control input gain matrix.

(2) In $[3,5,8,11,13,16,19,28-30]$, extra care should be taken to ensure that $\hat{G}\left(x, \theta_{g}\right)$ (recall that $\hat{G}\left(x, \theta_{g}\right)$ is the fuzzy estimate of $G(x)$ ) does not singular during the adaptation. However, in the proposed method, no extra care is need.

(3) An adaptation PI law augmented by e-modification is also proposed to update the fuzzy parameters.

The third fuzzy adaptive control scheme proposed, which is an indirect version, is developed for a class of MIMO unknown nonlinear systems with nonsymmetric or symmetric control gain matrix and with unknown sign. To the best of our knowledge, in the literature, there are only two works dealing with uncertain MIMO nonlinear systems with 
unknown sign of high-frequency gains [33,34]. The main contributions of this paper with respect to [33,34] are the following:

(1) The class of MIMO nonlinear systems is different from that considered in [33,34]. The modelling assumptions are realistic and not restrictive. Recall that in $[33,34]$, restrictive assumptions are made on the high-frequency control gains, i.e. the $g_{i i}(x)$ are assumed bounded. Moreover, to facilitate the stability analysis and the control design, the authors considered MIMO nonlinear systems with a lower triangular control structure.

(2) A unique Nussbaum-type function is used here in order to estimate the true sign of the control gain matrix. Note that in $[33,34]$ many Nussbaum-type functions have been used to deal with the unknown sign of control gains.

(3) Motivated by a matrix decomposition introduced in $[6,7,12,35]$, the control gain matrix $G(x)$ is decomposed into the product of a symmetric positive-definite matrix, a diagonal matrix with +1 or -1 on the diagonal and a unity upper triangular matrix.

(4) In our fuzzy adaptive control scheme, the method of stability analysis is relatively simple and different from that pursued in [33,34]. Recall that an integral Lyapunov function is considered in [33,34] in order to avoid the controller singularity which may be caused by time-varying gains function in indirect adaptive control schemes.

\section{Notation and problem statement}

Consider the following class of nonlinear MIMO systems described by

$$
\begin{aligned}
& y_{1}^{\left(r_{1}\right)}=f_{1}(x)+\sum_{j=1}^{p} g_{1 j}(x) u_{j}, \\
& \vdots \\
& y_{p}^{\left(r_{p}\right)}=f_{p}(x)+\sum_{j=1}^{p} g_{p j}(x) u_{j},
\end{aligned}
$$

where $x=\left[y_{1}, \dot{y}_{1}, \ldots, y_{1}^{\left(r_{1}-1\right)}, \ldots, y_{p}, \dot{y}_{p}, \ldots, y_{p}^{\left(r_{p}-1\right)}\right]^{T} \in R^{r}$ is the overall state vector which is assumed available for measurement and $r_{1}+\cdots+r_{p}=r, u=\left[u_{1}, \ldots, u_{p}\right]^{T} \in R^{p}$ is the control input vector, $y=\left[y_{1}, \ldots, y_{p}\right]^{T} \in R^{p}$ is the output vector, and $f_{i}(x), i=1, \ldots, p$ are continuous unknown nonlinear functions, and $g_{i j}(x), i, j=1, \ldots, p$ are continuous unknown nonlinear $C^{1}$ functions.

Let us denote

$$
\begin{aligned}
& y^{(r)}=\left[\begin{array}{lll}
y_{1}^{\left(r_{1}\right)} & \ldots & y_{p}^{\left(r_{p}\right)}
\end{array}\right]^{T}, \\
& F(x)=\left[\begin{array}{lll}
f_{1}(x) & \ldots & f_{p}(x)
\end{array}\right]^{T}, \\
& G(x)=\left[\begin{array}{ccc}
g_{11}(x) & \ldots & g_{1 p}(x) \\
\vdots & \ddots & \vdots \\
g_{p 1}(x) & \ldots & g_{p p}(x)
\end{array}\right] .
\end{aligned}
$$

Then, the system (1) can be rewritten in the following compact form:

$$
y^{(r)}=F(x)+G(x) u,
$$

where $F(\cdot) \in R^{p}$ and $G(\cdot) \in R^{p \times p}$.

The objective of this paper is to design a control law $u$ such as the output vector follows the specified desired trajectory $y_{d}=\left[y_{d 1}, \ldots, y_{d p}\right]^{T} \in R^{p}$, with all involved signals in the closed-loop system remain bounded.

Note that the desired trajectory vector, $x_{d}=\left[y_{d 1}, \dot{y}_{d 1}, \ldots, y_{d 1}^{\left(r_{1}-1\right)}, y_{d 1}^{\left(r_{1}\right)}, \ldots, y_{d p}, \dot{y}_{d p}, \ldots, y_{d p}^{\left(r_{p}-1\right)}, y_{d p}^{\left(r_{p}\right)}\right]^{T}$, is supposed continuous, bounded and available for measurement. Then, $x_{d} \in \Omega_{x_{d}} \subset R^{r+p}$, with $\Omega_{x_{d}}$ is a known bounded compact set. 
Let us define the tracking error as

$$
\begin{aligned}
& e_{1}=y_{d 1}-y_{1}, \\
& \vdots \\
& e_{p}=y_{d p}-y_{p}
\end{aligned}
$$

and the filtered tracking error as

$$
S=\left[S_{1}, \ldots, S_{p}\right]^{T},
$$

with

$$
S_{i}=\left[\frac{d}{d t}+\lambda_{i}\right]^{r_{i}-1} e_{i} \text { for } \lambda_{i}>0, \forall i=1, \ldots, p .
$$

Then, we can write (5) as follows:

$$
S_{i}=\lambda_{i}^{r_{i}-1} e_{i}+\left(r_{i}-1\right) \lambda_{i}^{r_{i}-2} \dot{e}_{i}+\cdots+\left(r_{i}-1\right) \lambda_{i} e_{i}^{\left(r_{i}-2\right)}+e_{i}^{\left(r_{i}-1\right)}
$$

with $i=1, \ldots, p$.

Notice that if we choose $\lambda_{i}>0$, with $i=1, \ldots, p$, then the roots of polynomial $H_{i}(s)=\lambda_{i}^{r_{i}-1}+\left(r_{i}-1\right) \lambda_{i}^{r_{i}-2} s+$ $\cdots+\left(r_{i}-1\right) \lambda_{i} s^{r_{i}-2}+s^{r_{i}-1}$ related to the characteristic equation of $S_{i}=0$ are all in the open left-half plane.

The relation (6) can be rewritten in the following compact form:

$$
S_{i}=C_{i}^{T} E_{i}
$$

with

$$
\begin{aligned}
& E_{i}=\left[\begin{array}{lllll}
e_{i} & \dot{e}_{i} & \cdots & e_{i}^{\left(r_{i}-2\right)} & e_{i}^{\left(r_{i}-1\right)}
\end{array}\right]^{T}, \\
& C_{i}^{T}=\left[\begin{array}{lll}
\lambda_{i}^{r_{i}-1} & \left(r_{i}-1\right) \lambda_{i}^{r_{i}-2} \ldots\left(r_{i}-1\right) \lambda_{i} 1
\end{array}\right] .
\end{aligned}
$$

Consequently, the vector $S$ takes the form:

$$
S=C^{T} E,
$$

where

$$
\begin{aligned}
C^{T} & =\operatorname{diag}\left[\begin{array}{llll}
C_{1}^{T} & C_{2}^{T} & \cdots & C_{p}^{T}
\end{array}\right]_{(p \times r)} \\
E & =\left[\begin{array}{llll}
E_{1}^{T} & E_{2}^{T} & \cdots & E_{p}^{T}
\end{array}\right]_{(r \times 1)}^{T}
\end{aligned}
$$

And the dynamic of $S_{i}$ is described by

$$
\dot{S}_{i}=C_{r i}^{T} E_{i}+e_{i}^{\left(r_{i}\right)} \text { and } i=1, \ldots, p,
$$

where $C_{r i}$ is given by

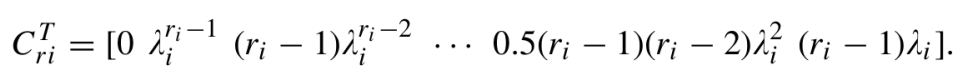

The dynamic of $S$ can be written into the following compact form:

$$
\dot{S}=C_{r}^{T} E+e^{(r)},
$$

where

$$
\begin{aligned}
C_{r}^{T} & =\operatorname{diag}\left[\begin{array}{llll}
C_{r 1}^{T} & C_{r 2}^{T} & \cdots & C_{r p}^{T}
\end{array}\right]_{(p \times r)}, \\
e^{(r)} & =\left[\begin{array}{llll}
e_{1}^{\left(r_{1}\right)} & e_{2}^{\left(r_{2}\right)} & \cdots & e_{p}^{\left(r_{p}\right)}
\end{array}\right]^{T},
\end{aligned}
$$


with

$$
e^{(r)}=y_{d}^{(r)}-y^{(r)}
$$

where $y^{(r)}=\left[\begin{array}{llll}y_{1}^{\left(r_{1}\right)} & y_{2}^{\left(r_{2}\right)} & \ldots & y_{p}^{\left(r_{p}\right)}\end{array}\right]^{T}$ is previously defined, and

$$
y_{d}^{(r)}=\left[\begin{array}{llll}
y_{d 1}^{\left(r_{1}\right)} & y_{d 2}^{\left(r_{2}\right)} & \cdots & y_{d p}^{\left(r_{p}\right)}
\end{array}\right]^{T} .
$$

From (18), we can write (15) as follows:

$$
\dot{S}=C_{r}^{T} E+y_{d}^{(r)}-y^{(r)} .
$$

Thereafter, (20) will be intensively used in the development of the fuzzy controllers and the stability analysis.

\section{Description of the fuzzy logic system}

The basic configuration of a fuzzy logic system consists of a fuzzifier, some fuzzy IF-THEN rules, a fuzzy inference engine and a defuzzifier, as shown in Fig. 1. The fuzzy inference engine uses the fuzzy IF-THEN rules to perform a mapping from an input vector $\underline{x}^{T}=\left[x_{1}, x_{2}, \ldots, x_{n}\right] \in R^{n}$ to an output $\hat{f} \in R$.

The $i$ th fuzzy rule is written as

$$
R^{(i)} \text { : if } x_{1} \text { is } A_{1}^{i} \text { and } \ldots \text { and } x_{n} \text { is } A_{n}^{i} \text { then } \hat{f} \text { is } f^{i},
$$

where $A_{1}^{i}, A_{2}^{i}, \ldots$, and $A_{n}^{i}$ are fuzzy sets and $f^{i}$ is the fuzzy singleton for the output in the $i$ th rule. By using the singleton fuzzifier, product inference, and center-average defuzzifier, the output of the fuzzy system can be expressed as follows:

$$
\begin{aligned}
\hat{f}(\underline{x}) & =\frac{\sum_{i=1}^{m} f^{i}\left(\prod_{j=1}^{n} \mu_{A_{j}^{i}}\left(x_{j}\right)\right)}{\sum_{i=1}^{m}\left(\prod_{j=1}^{n} \mu_{A_{j}^{i}}\left(x_{j}\right)\right)} \\
& =\theta^{T} \psi(\underline{x}),
\end{aligned}
$$

where $\mu_{A_{j}^{i}}\left(x_{j}\right)$ is the degree of membership of $x_{j}$ to $A_{j}^{i}, m$ is the number of fuzzy rules, $\theta^{T}=\left[f^{1}, f^{2}, \ldots, f^{m}\right]$ is the adjustable parameter vector (composed of consequent parameters), and $\psi^{T}=\left[\begin{array}{llll}\psi^{1} & \psi^{2} & \cdots & \psi^{m}\end{array}\right]$, where

$$
\psi^{i}(\underline{x})=\frac{\left(\prod_{j=1}^{n} \mu_{A_{j}^{i}}\left(x_{j}\right)\right)}{\sum_{i=1}^{m}\left(\prod_{j=1}^{n} \mu_{A_{j}^{i}}\left(x_{j}\right)\right)}
$$

is the fuzzy basis function (FBF). It is assumed that the FBFs are selected so that there is always at least one active rule [31], i.e. $\sum_{i=1}^{m}\left(\prod_{j=1}^{n} \mu_{A_{j}^{i}}\left(x_{j}\right)\right)>0$.

It is worth noting that the fuzzy system (22) is the most frequently used in control applications. Following the universal approximation results [31], the fuzzy system (22) is able to approximate any nonlinear smooth function $f$

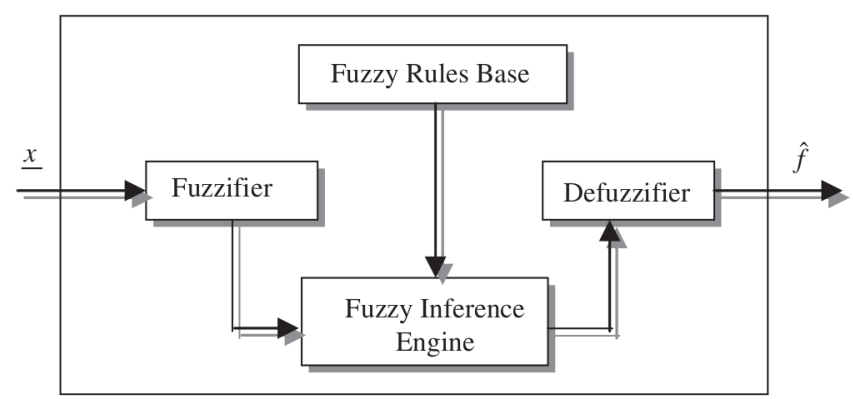

Fig. 1. The basic configuration of a fuzzy logic system. 
on a compact operating space to an arbitrary degree of accuracy. Of particular importance, it is assumed that the structure of the fuzzy system and the membership function parameters are properly specified in advance by the designer. This means that the designer decision is needed to determine the structure of the fuzzy system, namely the pertinent inputs, the number of membership functions for each input, the membership function parameters and the number of rules. As for the consequent parameters, i.e. $\theta$, they must be determined by learning algorithms.

\section{Design of fuzzy adaptive controllers}

In this section, three fuzzy adaptive control schemes are developed for three different classes of unknown nonlinear systems, namely:

- MIMO systems with $G(x)$ symmetric and of a known sign,

- MIMO systems with known signs of the leading principal minors of $G(x)$, but it can be symmetric or nonsymmetric,

- MIMO systems with an unknown sign of $G(x)$, but it can be symmetric or nonsymmetric.

\subsection{First fuzzy adaptive control scheme}

In this section, we will develop a direct fuzzy adaptive control where the control gain matrix is supposed symmetric and with known sign. In the controller design, we need the following assumptions on system (2).

\section{Assumption 1.}

(a) $G(x)$ is symmetric positive-definite. Then, it exists an unknown positive constant $\sigma_{0}$, such that: $G(x) \geq \sigma_{0} I_{p}$, with $I_{P}$ is an identity matrix.

(b)

$$
\frac{1}{2}\left\|\dot{G}^{-1}(x)\right\| \leq \beta(x), \quad \forall x \in R^{r},
$$

where $\beta(x)$ is an unknown continuous positive function.

\section{Remark 1.}

(a) There are many physical systems, such as robotic systems and electrical machines, which satisfy Assumption 1(a). In fact, this assumption enables us to have:

- an easy analysis of the stability,

- and especially, such a property of $G(x)$ ensures that the matrix $G(x)$ is always regular and, therefore system (1) is feedback linearizable by a static state feedback.

(b) Assumption 1(b) is not restrictive, since we assume only the existence of $\beta(x)$ and not its knowledge. Moreover, there are several physical (MIMO or SISO) systems of which the control gain matrix $G(x)$ satisfies the inequality (24), e.g. manipulator robots, electrical machines, inverted pendulum, chaotic systems, etc. In fact, these dynamical nonlinear systems and many others are characterized by a control gain matrix which depends only on the vector $x_{g}=\left[y_{1}, \dot{y}_{1}, \ldots, y_{1}^{\left(r_{1}-2\right)}, \ldots, y_{p}, \dot{y}_{p}, \ldots, y_{p}^{\left(r_{p}-2\right)}\right]^{T} \in R^{r-p}$, then the matrix $\dot{G}^{-1}(x)$ depends only on the state vector $x=\left[y_{1}, \dot{y}_{1}, \ldots, y_{1}^{\left(r_{1}-1\right)}, \ldots, y_{p}, \dot{y}_{p}, \ldots, y_{p}^{\left(r_{p}-1\right)}\right]^{T}$.

(c) To the best of our knowledge, in the literature, there are only four works dealing with direct fuzzy control for MIMO nonlinear systems $[3,14,16,19]$. Compared with our assumption, restrictive assumptions are made on the control gain matrix in $[3,14,16,19]$, namely:

- In [3], the control gain matrix $G(x)$ is assumed positive-definite and known.

- In [16], $G(x)$ is supposed positive-definite, constant and known.

- As for [19], each entry of $G(x)$ is bounded by known constant $\left|g_{i j}(x)\right| \leq \bar{g}_{i j}, i, j=1, \ldots, p, i \neq j$. Also, the entries in main diagonal must satisfy $0<\underline{g}_{i j} \leq g_{i i}(x) \leq \bar{g}_{i j}<\infty, i=1, \ldots, p$ and their derivatives be defined and satisfy $\left|\dot{g}_{i i}(x)\right| \leq M_{i i}(x), i=1, \ldots, p$, where $\underline{g}_{i j}, \bar{g}_{i j}$ and $M_{i i}(x)$ are known bounds. 
- In [14], $G(x)$ is assumed symmetric positive-definite and bounded as follows: $0_{p \times p}<\sigma_{0} I_{p} \leq G(x) \leq \sigma_{1} I_{p}$, where $\sigma_{0}$ and $\sigma_{1}$ are some positive constants.

Substituting (2) into the tracking error dynamics (20), we get

$$
\dot{S}=C_{r}^{T} E+y_{d}^{(r)}-F(x)-G(x) u .
$$

Eq. (25) can be arranged as follows:

$$
G_{1}(x) \dot{S}=G_{1}(x) v+F_{1}(x)-u,
$$

where $G_{1}(x)=G^{-1}(x), F_{1}(x)=-G^{-1}(x) F(x)$ and the vector $v$ is given by

$$
v=C_{r}^{T} E+y_{d}^{(r)}
$$

It is very clear that if $G(x)$ is symmetric and positive-definite, then its inverse $G_{1}(x)=G^{-1}(x)$ is also symmetric and positive-definite. In fact, this useful property of $G_{1}(x)$ will be intensively exploited in the stability analysis.

\subsubsection{Ideal controller}

If the nonlinear functions $G(x)$ and $F(x)$ (or $G_{1}(x)$ and $F_{1}(x)$ ) are known, to achieve the control objectives, we can use the following ideal control $u^{*}$ :

$$
u=u^{*}=G_{1}(x) v+F_{1}(x)+\left(K+\beta(x) I_{p}\right) S,
$$

where $\beta(x)$ is previously defined in Assumption 1(b), $K$ is a positive diagonal matrix.

If we apply the ideal controller (28) to system (2) (i.e. if we replace the ideal controller (28) in error dynamics (26)), we obtain the following closed-loop system dynamics:

$$
G_{1}(x) \dot{S}=-\left(K+\beta(x) I_{p}\right) S .
$$

Theorem 1. Consider system (1). Suppose that Assumption 1 is satisfied. Then, the control law defined by (28) guarantees the following properties:

- All signals in the closed loop system are bounded.

- The tracking errors and their derivatives decrease asymptotically to zero, i.e. $e_{i}^{(j)}(t) \rightarrow 0$ as $t \rightarrow \infty$ for $i=1, \ldots, p$ and $j=0,1, \ldots, r_{i}-1$.

Proof of Theorem 1. Since $G_{1}(x)$ is symmetric positive-definite, we can define the following Lyapunov function:

$$
V=\frac{1}{2} S^{T} G_{1}(x) S .
$$

The time derivative of $V$ is given by

$$
\dot{V}=S^{T} G_{1}(x) \dot{S}+\frac{1}{2} S^{T} \dot{G}_{1}(x) S .
$$

Using (24) and (29), (31) can be bounded as follows:

$$
\begin{aligned}
\dot{V} & =S^{T} G_{1}(x) \dot{S}+\frac{1}{2} S^{T} \dot{G}_{1}(x) S \\
& \leq-S^{T}(K+\beta(x) I) S+\frac{1}{2}\left\|\dot{G}_{1}(x)\right\|\|S\|^{2} \\
& \leq-S^{T} K S .
\end{aligned}
$$

From (32), and using the Barbalat's lemma, we can easily show, as in [22], that $s_{i} \rightarrow 0$, when $t \rightarrow \infty$. Thus, the tracking errors and their derivatives decrease asymptotically to zero, i.e. $e_{i}^{(j)}(t) \rightarrow 0$ as $t \rightarrow \infty$ for $i=1, \ldots, p$ and $j=0,1, \ldots, r_{i}-1$. 


\subsubsection{Fuzzy adaptive control design and stability analysis}

Since the nonlinear functions $G(x)$ and $F(x)$ are not known, the above controller, i.e. (28), cannot be applied directly. In this paper, we propose the use of fuzzy systems to approximate the ideal controller (28).

Before proposing an estimation scheme for the ideal controller, let us rearrange (28) as follows:

$$
\begin{aligned}
u^{*} & =G_{1}(x) v+F_{1}(x)+\left(K+\beta(x) I_{p}\right) S \\
& =\alpha(\bar{x})+K S,
\end{aligned}
$$

where $\alpha(\bar{x})=\left[\alpha_{1}(\bar{x}), \alpha_{2}(\bar{x}), \ldots, \alpha_{p}(\bar{x})\right]^{T}=G_{1}(x) v+F_{1}(x)+\beta(x) I_{p} S$, with $\bar{x}=\left[\begin{array}{lll}x^{T} & v^{T} & S^{T}\end{array}\right]^{T} \in D_{\bar{x}} \subset R^{r} \times R^{p} \times R^{p}$. The compact set $D_{\bar{x}}$ is defied as follows:

$$
\Omega_{\bar{x}}=\left\{\left[x^{T}, v^{T}, S^{T}\right] \mid x \in \Omega_{x} \subset R^{r}, x_{d} \in \Omega_{x_{d}}\right\}
$$

Thereafter, the fuzzy estimate of $\alpha(\bar{x})$ is used to develop an adaptive controller with its adaptive law in order to meet control objectives and guarantee boundedness of all involved signals of the closed-loop system.

The nonlinear function $\alpha_{i}(\bar{x})$ can be approximated, over compact set $D_{\bar{x}}$, by the fuzzy systems (22) as follows:

$$
\hat{\alpha}_{i}\left(\bar{x} \theta_{i}\right)=\theta_{i}^{T} \psi_{i}(\bar{x}), \quad i=1, \ldots, p,
$$

where $\psi_{i}(\bar{x})$ is the FBF vector, which is fixed a priori by the designer, and $\theta_{i}$ is the adjustable parameter vector of the fuzzy system.

Let us define

$$
\theta_{i}^{*}=\underset{\theta_{i}}{\arg \min }\left[\sup _{\bar{x} \in D_{\bar{x}}}\left|\alpha_{i}(\bar{x})-\hat{\alpha}_{i}\left(\bar{x}, \theta_{i}\right)\right|\right]
$$

as the ideal parameters of $\theta_{i}$.

Note that the ideal parameters $\theta_{i}^{*}$ are artificial constant quantities introduced only for analysis purposes, and their values are not needed when implementing the controller.

Define

$$
\tilde{\theta}_{i}=\theta_{i}-\theta_{i}^{*} \text { with } i=1, \ldots, p
$$

as the parameter estimation error, and

$$
\varepsilon_{i}(\bar{x})=\alpha_{i}(\bar{x})-\hat{\alpha}_{i}\left(\bar{x}, \theta_{i}^{*}\right)
$$

is the fuzzy approximation error, where $\hat{\alpha}_{i}\left(\bar{x}, \theta_{i}^{*}\right)=\theta_{i}^{* T} \psi_{i}(\bar{x})$.

As in literature [31], we commonly assume that the used fuzzy systems do not violate the universal approximator property on the compact set $D_{\bar{x}}$, which is assumed large enough so that input vector of the fuzzy system remains within $D_{\bar{x}}$ under closed-loop control system. So it is reasonable to assume that the fuzzy approximation error is bounded for all $\bar{x} \in D_{\bar{x}}$, i.e.

$$
\left|\varepsilon_{i}(\bar{x})\right| \leq \bar{\varepsilon}_{i}, \quad \forall \bar{x} \in D_{\bar{x}},
$$

where $\bar{\varepsilon}_{i}$ is a given constant.

Now, let us denote

$$
\begin{aligned}
& \hat{\alpha}(\bar{x}, \theta)=\theta^{T} \psi(\bar{x})=\left[\hat{\alpha}_{1}\left(\bar{x}, \theta_{1}\right), \ldots, \hat{\alpha}_{p}\left(\bar{x}, \theta_{p}\right)\right]^{T}, \\
& \varepsilon(\bar{x})=\left[\varepsilon_{1}(\bar{x}), \ldots, \varepsilon_{p}(\bar{x})\right]^{T}, \\
& \bar{\varepsilon}=\left[\bar{\varepsilon}_{1}, \ldots, \bar{\varepsilon}_{p}\right]^{T} .
\end{aligned}
$$

From the above analysis, we have

$$
\begin{aligned}
\hat{\alpha}(\bar{x}, \theta)-\alpha(\bar{x}) & =\hat{\alpha}(\bar{x}, \theta)-\hat{\alpha}\left(\bar{x}, \theta^{*}\right)+\hat{\alpha}\left(\bar{x}, \theta^{*}\right)-\alpha(\bar{x}) \\
& =\hat{\alpha}(\bar{x}, \theta)-\hat{\alpha}\left(\bar{x}, \theta^{*}\right)-\varepsilon(\bar{x}) \\
& =\tilde{\theta}^{T} \psi(\bar{x})-\varepsilon(\bar{x}),
\end{aligned}
$$

where $\tilde{\theta}^{T} \psi(\bar{x})=\left[\tilde{\theta}_{1}^{T} \psi_{1}(\bar{x}), \tilde{\theta}_{2}^{T} \psi_{2}(\bar{x}), \ldots, \tilde{\theta}_{p}^{T} \psi_{p}(\bar{x})\right]^{T}$, and $\tilde{\theta}_{i}=\theta_{i}-\theta_{i}^{*}, i=1, \ldots, p$. 
We can rearrange the tracking error dynamics (26) as follows:

$$
G_{1}(x) \dot{S}=\alpha(\bar{x})-u-\beta(x) I_{p} S .
$$

Recall that $\alpha(\bar{x})=\left[\alpha_{1}(\bar{x}), \alpha_{2}(\bar{x}), \ldots, \alpha_{p}(\bar{x})\right]^{T}=G_{1}(x) v+F_{1}(x)+\beta(x) I_{p} S$.

Using (34), the ideal control (33) can be approximated as follows:

$$
\begin{aligned}
u & =\hat{\alpha}(\bar{x}, \theta)+K_{1} S+K_{0} \operatorname{Sign}(S) \\
& =\theta^{T} \psi(\bar{x})+K_{1} S+K_{0} \operatorname{Sign}(S),
\end{aligned}
$$

where $\hat{\alpha}(\bar{x}, \theta)=\theta^{T} \psi(\bar{x})=\left[\theta_{1}^{T} \psi_{1}(\bar{x}), \theta_{2}^{T} \psi_{2}(\bar{x}), \ldots, \theta_{p}^{T} \psi_{p}(\bar{x})\right]^{T}$, and $K_{0}=\operatorname{diag}\left[k_{01}, k_{02}, \ldots, k_{0 p}\right]$, with $k_{0 i}>0, i=$ $1, \ldots, p$, are positive constants and will be defined later. $K_{1}=\operatorname{diag}\left[k_{11}, k_{12}, \ldots, k_{1 p}\right]$, with $k_{1 i}>0, i=1, \ldots, p$, are free positive constants of the design.

By applying the controller (39) to system (2) (i.e. by replacing (39) into tracking error dynamics (38)), and using expression (37), we can obtain

$$
G_{1}(x) \dot{S}=-\beta(x) I_{p} S-K_{1} S-K_{0} \operatorname{Sign}(S)-\tilde{\theta}^{T} \psi(\bar{x})+\varepsilon(\bar{x}) .
$$

Multiplying $S^{T}$ to (40), we have

$$
S^{T} G_{1}(x) \dot{S}=-\beta(x)\|S\|^{2}-S^{T} K_{1} S-\sum_{i=1}^{p} K_{0 i}\left|S_{i}\right|-\sum_{i=1}^{p} \tilde{\theta}_{i}^{T} \psi_{i}(\bar{x}) S_{i}+S^{T} \varepsilon(\bar{x}) .
$$

In order to meet the control objective, the fuzzy parameters $\theta_{i}$ are updated by the following adaptation PI law:

$$
\theta_{i}=\int_{0}^{t}\left[-\sigma_{i} \gamma_{0 i}\left|S_{i}\right| \theta_{i}+\gamma_{0 i} S_{i} \psi_{i}(\bar{x})\right] d \tau-\gamma_{1 i} \delta_{i},
$$

with

$$
\delta_{i}=\sigma_{i}\left|S_{i}\right| \theta_{i}-S_{i} \psi_{i}(\bar{x}),
$$

where $\gamma_{0 i}, \gamma_{1 i}, \sigma_{i}>0$ are design constants. It is worth mentioning that, in the adaptation law (42), two terms are introduced for different purposes. The term $\sigma_{i} \gamma_{0 i}\left|S_{i}\right| \theta_{i}$, borrowed to the e-modification concept [17], is mainly motivated by parameter boundedness purposes. Unlike $\sigma$-modification concept, the combination of e-modification concept with the robust term $K_{0} \operatorname{Sign}(S)$ in the proposed adaptive control schemes can assure a convergence of the tracking error to zero. Whereas $\delta_{i}$, which is a proportional term, can allow to fuzzy adaptive parameters a fast convergence. Indeed, afterwards, we will see that the addition of the latter to the adaptation law (42) makes possible to have an important negative term in the Lyapunov's function derivative.

Note that the control term $\theta^{T} \psi(\bar{x})+K_{1} S$ in (39) is an estimation of the ideal controller (33). As for the last term $K_{0} \operatorname{Sign}(S)$, it is used to deal with the fuzzy approximation error and external disturbances and eliminate the effect of terms $0.5 \sigma_{i}\left\|\theta_{i}^{*}\right\|^{2}, i=1, \ldots, p$, due to the use of the $e$-modificationin the adaptation law (42).

In summary, the update law (42) has a nice property as stated by the following lemma.

Lemma 1. The adaptation PI law (42) guarantees that $\theta_{i} \in L_{\infty}$ for bounded initial parameters $\theta_{i}(0)$.

Proof of Lemma 1. Choose the following Lyapunov candidate function:

$$
V_{\theta_{i}}=\frac{1}{2 \gamma_{0 i}}\left(\theta_{i}+\gamma_{1 i} \delta_{i}\right)^{T}\left(\theta_{i}+\gamma_{1 i} \delta_{i}\right) .
$$

The time derivative of $V_{\theta_{i}}$ along of (42) is

$$
\begin{aligned}
\dot{V}_{\theta_{i}} & =\frac{1}{\gamma_{0 i}}\left(\theta_{i}+\gamma_{1 i} \delta_{i}\right)^{T}\left(\dot{\theta}_{i}+\gamma_{1 i} \dot{\delta}_{i}\right) \\
& =\left(\theta_{i}+\gamma_{1 i} \delta_{i}\right)^{T}\left(-\sigma_{i}\left|S_{i}\right| \theta_{i}+S_{i} \psi_{i}(\bar{x})\right) .
\end{aligned}
$$


If we select $\delta_{i}=\sigma_{i}\left|S_{i}\right| \theta_{i}-S_{i} \psi_{i}(\bar{x})$, we get

$$
\begin{aligned}
\dot{V}_{\theta_{i}} & \leq-\sigma_{i}\left|S_{i}\right|\left\|\theta_{i}\right\|^{2}+\left|S_{i}\right|\left\|\theta_{i}\right\|\left\|\psi_{i}(\bar{x})\right\|-\gamma_{1 i}\left\|\delta_{i}\right\|^{2} \\
& \leq-\sigma_{i}\left|S_{i}\right|\left\|\theta_{i}\right\|\left(\left\|\theta_{i}\right\|-\left(c_{1 i} / \sigma_{i}\right)\right) .
\end{aligned}
$$

Noting $c_{1 i}=\sup _{t}\left\|\psi_{i}(\bar{x})\right\|$. Therefore $\dot{V}_{\theta_{i}}<0$ once $\left\|\theta_{i}\right\|>c_{1 i} / \sigma_{i}$. Because $c_{1 i}$ and $\sigma_{i}$ are positive constants, one concludes that $\theta_{i} \in L_{\infty}$.

Remark 2. From (45), we remark that $\delta_{i}$ introduces in $\dot{V}_{\theta_{i}}$ a negative term which can be important, if $\gamma_{1 i}$ is selected large. Consequently, the proportional term $\delta_{i}$ can contribute to the closed-loop stability and can allow to $\theta_{i}$ a fast convergence.

Remark 3. In order to avoid the problem of the algebraic loop being in (42), when implementing the controller, the update law (42) must be rearranged as follows:

$$
\theta_{i}=\frac{1}{1+\gamma_{1 i} \sigma_{i}\left|S_{i}\right|}\left(\int_{0}^{t}\left[-\sigma_{i} \gamma_{0 i}\left|S_{i}\right| \theta_{i}+\gamma_{0 i} S_{i} \psi_{i}(\bar{x})\right] d \tau+\gamma_{1 i} S_{i} \psi_{i}(\bar{x})\right)
$$

Now, we are ready to give the following theorem:

Theorem 2. Consider system (1). Suppose that Assumption 1 is satisfied. Then, the control law defined by (39) with the adaptation law given by (42) guarantee the following properties:

- All signals in the closed-loop system are bounded.

- The tracking errors and their derivatives decrease asymptotically to zero, i.e. $e_{i}^{(j)}(t) \rightarrow 0$ as $t \rightarrow \infty$ for $i=1, \ldots, p$ and $j=0,1, \ldots, r_{i}-1$.

Proof of Theorem 2. Let us consider the following Lyapunov function candidate:

$$
V=\frac{1}{2} S^{T} G_{1}(x) S+\frac{1}{2} \sum_{i=1}^{p} \frac{1}{\gamma_{0 i}}\left(\tilde{\theta}_{i}+\gamma_{1 i} \delta_{i}\right)^{T}\left(\tilde{\theta}_{i}+\gamma_{1 i} \delta_{i}\right) .
$$

Its time derivative is given by

$$
\dot{V}=S^{T} G_{1}(x) \dot{S}+\frac{1}{2} S^{T} \dot{G}_{1}(x) S+\sum_{i=1}^{p} \frac{1}{\gamma_{0 i}}\left(\tilde{\theta}_{i}+\gamma_{1 i} \delta_{i}\right)^{T}\left(\dot{\theta}_{i}+\gamma_{1 i} \dot{\delta}_{i}\right) .
$$

Eq. (47) can be expressed as

$$
\dot{V}=\dot{V}_{1}+\dot{V}_{2},
$$

where

$$
\dot{V}_{1}=S^{T} G_{1}(x) \dot{S}+\frac{1}{2} S^{T} \dot{G}_{1}(x) S
$$

and

$$
\dot{V}_{2}=\sum_{i=1}^{p} \frac{1}{\gamma_{0 i}}\left(\tilde{\theta}_{i}+\gamma_{i 1} \delta_{i}\right)^{T}\left(\dot{\theta}_{i}+\gamma_{1 i} \dot{\delta}_{i}\right)
$$

Using (41) and Assumption 1, $\dot{V}_{1}$ can be bounded as follows:

$$
\begin{aligned}
\dot{V}_{1} & \leq-S^{T} K_{1} S-\sum_{i=1}^{p} K_{0 i}\left|S_{i}\right|-\beta(x)\|S\|^{2}+0.5\|\dot{G}(x)\|\|S\|^{2}-\sum_{i=1}^{p} \tilde{\theta}_{i}^{T} \psi_{i}(\bar{x}) S_{i}+S^{T} \varepsilon(\bar{x}) \\
& \leq-S^{T} K_{1} S-\sum_{i=1}^{p} K_{0 i}\left|S_{i}\right|-\sum_{i=1}^{p} \tilde{\theta}_{i}^{T} \psi_{i}(\bar{x}) S_{i}+\sum_{i=1}^{p} \bar{\varepsilon}_{i}\left|S_{i}\right| .
\end{aligned}
$$


The substitution of the parameter adaptation law (42) into (50) gives

$$
\begin{aligned}
\dot{V}_{2} & =\sum_{i=1}^{p}\left(\tilde{\theta}_{i}+\gamma_{1 i} \delta_{1 i}\right)^{T}\left(-\sigma_{i}\left|S_{i}\right| \theta_{i}+S_{i} \psi_{i}(\bar{x})\right) \\
& \leq \sum_{i=1}^{p} \tilde{\theta}_{i}^{T} \psi_{i}(\bar{x}) S_{i}-\sum_{i=1}^{p} \gamma_{1 i}\left\|\delta_{1 i}\right\|^{2}-\frac{1}{2} \sum_{i=1}^{p} \sigma_{i}\left|S_{i}\right|\left\|\tilde{\theta}_{i}\right\|^{2}+\frac{1}{2} \sum_{i=1}^{p} \sigma_{i}\left|S_{i}\right|\left\|\theta_{i}^{*}\right\|^{2},
\end{aligned}
$$

where the following inequality $-2 \tilde{\theta}_{i}^{T} \theta_{i} \leq-\sum_{i=1}^{p}\left\|\tilde{\theta}_{i}\right\|^{2}+\sum_{i=1}^{p}\left\|\theta_{i}^{*}\right\|^{2}$ was used in the above development.

From the previous results, it follows that

$$
\dot{V} \leq-\sum_{i=1}^{p} k_{1 i} S_{i}^{2}-\sum_{i=1}^{p} k_{0 i}\left|S_{i}\right|+\sum_{i=1}^{p} \bar{\varepsilon}_{i}\left|S_{i}\right|+0.5 \sum_{i=1}^{p} \sigma_{i}\left|S_{i}\right|\left\|\theta_{i}^{*}\right\|^{2} .
$$

If we select $k_{0 i} \geq 0.5 \sum_{i=1}^{p} \sigma_{i}\left\|\theta_{i}^{*}\right\|^{2}+\bar{\varepsilon}_{i}, \dot{V}$ can be bounded by

$$
\dot{V} \leq-\sum_{i=1}^{p} k_{1 i} S_{i}^{2}
$$

From (54), $\dot{V}$ is always negative, which implies that the signals $S_{i}$ and $\tilde{\theta}_{i}+\gamma_{1 i} \delta_{i}$ are bounded.

To show the boundedness of $\tilde{\theta}_{i}$ and $\theta_{i}$, we can use the following expression which is obtained from the expression of $\delta_{i}$ in Eq. (42) and $\tilde{\theta}_{i}=\theta_{i}-\theta_{i}^{*}$ :

$$
\theta_{i}=\frac{\left[\tilde{\theta}_{i}+\gamma_{1 i} \delta_{i}\right]+\gamma_{1 i} S_{i} \psi_{i}(\bar{x})+\theta_{i}^{*}}{1+\gamma_{1 i} \sigma_{i}\left|S_{i}\right|}
$$

Using the above expression, and because $\tilde{\theta}_{i}+\gamma_{1 i} \delta_{i}, \theta_{i}^{*}, \psi_{i}(\bar{x})$ and $S_{i} \in L_{\infty}$, we can easily show that $\theta_{i}$ and $\tilde{\theta}_{i} \in L_{\infty}$.

Since $S_{i}, \theta_{i} \in L_{\infty}$, it implies the boundedness of $\bar{x}, u$. Since $V$ is non-increasing function of time and bounded from below, the $\lim _{t \rightarrow \infty} V(t)=V(\infty)$ exists. By integrating (54) from 0 to $\infty$, we have

$$
\int_{0}^{\infty} \sum_{i=1}^{p} k_{1 i} S_{i}^{2}(t) d t \leq V(0)-V(\infty)<\infty
$$

which implies that $S_{i} \in L_{2}$.

In order to show the boundedness of $\dot{S}_{i}$, we must rearrange Eq. (40) as follows:

$$
\dot{S}=G(x)\left[-\beta(x) I_{p} S-K S-K_{0} \operatorname{Sign}(S)-\tilde{\theta}^{T} \psi(\bar{x})+\varepsilon(\bar{x})\right],
$$

where $G(x)=G_{1}^{-1}(x)$. Since $x \in L_{\infty}$, and the functions $G(x)$ and $\beta(x)$ are continuous functions, then they are bounded. Since all terms in the right of (56) are bounded, then $\dot{S}_{i} \in L_{\infty}$.

Finally, since $S_{i} \in L_{2} \cap L_{\infty}$ and $\dot{S}_{i} \in L_{\infty}$, by using Barbalat's lemma [22] we can conclude that $S_{i}(t) \rightarrow 0$ as $t \rightarrow \infty$. Therefore, the tracking errors and their derivatives converge asymptotically to zero, i.e. $e_{i}^{(j)}(t) \rightarrow 0$ as $t \rightarrow \infty$ for $i=1 \cdots p$ and $j=0,1, \ldots, r_{i}-1$.

\subsection{Second fuzzy adaptive control scheme}

The control scheme presented previously is only applicable for nonlinear systems with a standard, symmetric control gain matrix (e.g., Lagrange-Euler systems, electrical machines). It is worth noting that there are some practical systems which do not possess a symmetric control gain matrix $G(x)$, e.g. the visual servoing problem and the automotive thermal management system [35]. Motivated by a matrix decomposition introduced in [6,7,12], we can decompose $G(x)$ into the product of a symmetric positive-definite matrix $G_{s}(x) \in R^{P \times P}$ and a diagonal matrix $D \in R^{P \times P}$ with +1 or -1 on the diagonal (comprised the ratios of the signs of the leading principal minors of the control input gain matrix) and a unity upper triangular matrix $T(x) \in R^{P \times P}$. 


\subsubsection{Decomposition of the matrix $G(x)$}

The design of this fuzzy controller and the third controller (in Section 4.3) is based on the following useful lemma.

Lemma 2 (Costa et al. [7]). Any real matrix $G(x) \in R^{p \times p}$ with non-zero leading principal minors can be decomposed as follows:

$$
G(x)=G_{s}(x) D T(x),
$$

where $G_{s}(x) \in R^{p \times p}$ is a symmetric positive-definite matrix, $D \in R^{p \times p}$ is a diagonal matrix with +1 or -1 on the diagonal and $T(x) \in R^{p \times p}$ is a unity upper triangular.

Proof of Lemma 2. See [7,24].

It is worth noting that the decomposition of the matrix $G(x)$ in (57) is very useful. In fact, the symmetric positivedefinite matrix $G_{s}(x)$ will be exploited in the Lyapunov-based stability, $D$ contains information on the sign of the original matrix $G(x)$, while the unity upper triangular matrix $T(x)$ allows for algebraic loop free sequential synthesis of control signals $u_{i}, \forall i=1,2, \ldots, p$.

Note that if $G(x)$ has non-zero leading principal minors, three cases can arise:

- If $G(x)$ is positive-definite, then $D=I_{P}$.

- If $G(x)$ is negative-definite, then $D=-I_{p}$.

- While in the case where $G(x)$ is indefinite, the matrix $D$ has +1 and -1 on the diagonal.

\subsubsection{Fuzzy adaptive control design}

Consider system (2), with the following assumption.

\section{Assumption 2.}

(a) $G(x)$ has non-zero leading principal minors and their signs are also known.

(b) $G_{s}(x)$ and $(d / d t) G_{s}^{-1}(x)$ are continuous.

(c) $\partial g_{i j}(x) / \partial y_{i}^{\left(r_{i}-1\right)}=0, \forall i=1,2, \ldots, p$, and $j=1,2, \ldots, p$.

\section{Remark 4.}

(a) It is worth noting that several physical systems (MIMO or SISO) have a $G(x)$ positive-definite (i.e. $D=I_{P}$ ), namely: robotic systems, electrical machines, inverted pendulum. However, the assumption on the signs knowledge of the leading principal minors of $G(x)$ can be restrictive in the general case.

(b) Assumption 2(c) means that the control gain matrix $G(x)$ depends only on the following state vector $x_{g}=$ $\left[y_{1}, \dot{y}_{1}, \ldots, y_{1}^{\left(r_{1}-2\right)}, \ldots, y_{p}, \dot{y}_{p}, \ldots, y_{p}^{\left(r_{p}-2\right)}\right]^{T} \in R^{r-p}$. Consequently, matrices $G_{s}(x)$ and $T(x)$ are only functions of $x_{g}$. Physically speaking, Assumption 2(c) is not restrictive as there are several (MIMO or SISO) systems of which the control gain matrix $G(x)$ satisfies Assumption 2(c), e.g. manipulator robots, electrical machines, inverted pendulum, chaotic systems. Note that Assumption 2(c) allows us to have a $d G_{s}^{-1}(x) / d t$ which depends only on the state vector $x=\left[y_{1}, \dot{y}_{1}, \ldots, y_{1}^{\left(r_{1}-1\right)}, \ldots, y_{p}, \dot{y}_{p}, \ldots, y_{p}^{\left(r_{p}-1\right)}\right]^{T} \in R^{r}$.

Using the matrix composition (57) and the dynamics of (20), the dynamics of $S$ can be rewritten as follows:

$$
G_{s}^{-1}(x) \dot{S}=G_{s}^{-1}(x)[v-F(x)]-D T(x) u .
$$

Posing $G_{1}(x)=G_{s}^{-1}(x), F_{1}(x, u)=G_{s}^{-1}(x)[v-F(x)]-[D T(x)-D] u$, Eq. (58) becomes

$$
G_{1}(x) \dot{S}=F_{1}(x, u)-D u .
$$

Similar to the previous fuzzy adaptive control scheme, (59) can be rearranged as follows:

$$
G_{1}(x) \dot{S}=-\frac{1}{2} \dot{G}_{1} S+\alpha(\bar{z})-D u,
$$

where $\alpha(\bar{z})=\left[\alpha_{1}\left(\bar{z}_{1}\right), \alpha_{2}\left(\bar{z}_{2}\right), \ldots, \alpha_{p}\left(\bar{z}_{P}\right)\right]^{T}=F_{1}(x, u)+\frac{1}{2} \dot{G}_{1}(x) S$, with $\bar{z}=\left[\bar{z}_{1}^{T}, \bar{z}_{2}^{T}, \ldots, \bar{z}_{p}^{T}\right]^{T}$. 
By examining the expression of $F_{1}(x, u)$ and $\alpha(\bar{z})$, the elements of the vector $\bar{z}$ can be selected by

$$
\begin{aligned}
& \bar{z}_{1}=\left[x^{T}, S^{T}, u_{2}, \ldots, u_{p}\right]^{T}, \\
& \bar{z}_{2}=\left[x^{T}, S^{T}, u_{3}, \ldots, u_{p}\right]^{T}, \\
& \vdots \\
& \bar{z}_{p-1}=\left[x^{T}, S^{T}, u_{P}\right]^{T}, \\
& \bar{z}_{p}=\left[x^{T}, S^{T}\right]^{T} .
\end{aligned}
$$

It is very clear from the propriety of the matrix of $D T(x)-D$ that $\bar{z}_{1}$ depends on control inputs $u_{2}, \ldots, u_{p}, \bar{z}_{2}$ depends on $u_{3}, \ldots, u_{p}$, and so on. In fact, the structure of the nonlinearities $\alpha(\bar{z})$ is known under the name "upper triangular control structure". Recall that this useful structure allows for algebraic loop free sequential synthesis of control signals $u_{i}, \forall i=1,2, \ldots, p$.

Define the compact sets as follows:

$$
\begin{aligned}
& D_{\bar{z}_{i}}=\left\{\left[x^{T}, S^{T}, u_{i+1}, \ldots, u_{p}\right]^{T} \mid x \in \Omega_{x} \subset R^{r}, x_{d} \in \Omega_{x_{d}}\right\}, \quad i=1,2, \ldots, p-1, \\
& \Omega_{\bar{z}_{p}}=\left\{\left[x^{T}, S^{T}\right] \mid x \in \Omega_{x} \subset R^{r}, x_{d} \in \Omega_{x_{d}}\right\} .
\end{aligned}
$$

Remark 5. The choice of the vectors $\bar{z}_{i}$ (input arguments of the unknown functions $\alpha_{i}$ ) is not unique. In fact, since we known that $S$ and $u$ are functions of state $x$ and $x_{d}$, then it can be seen quite simply that all $\bar{z}_{i}$ are functions of $x$ and $x_{d}$ (e.g. we can choose $\bar{z}_{i}=\left[x^{T}, x_{d}^{T}\right]^{T}$ or $\bar{z}_{i}=\left[x^{T}, E^{T}\right]^{T}$, with $i=1,2, \ldots, p$ ). Also, since $x_{d}$ is bounded, we can choose $\bar{z}_{i}=x$.

The unknown smooth function $\alpha(\bar{z})$ can be approximated optimally by fuzzy logic systems as follows:

$$
\begin{aligned}
\alpha(\bar{z}) & =\hat{\alpha}\left(\bar{z}, \theta^{*}\right)+\varepsilon(\bar{z}) \\
& =\theta^{* T} \psi(\bar{z})+\varepsilon(\bar{z}),
\end{aligned}
$$

where $\theta^{* T} \psi(\bar{z})=\left[\theta_{1}^{* T} \psi_{1}\left(\bar{z}_{1}\right), \theta_{2}^{* T} \psi_{2}\left(\bar{z}_{2}\right), \ldots, \theta_{P}^{* T} \psi_{P}\left(\bar{z}_{P}\right)\right]^{T}$ and $\varepsilon\left(\bar{z}_{2}\right)=\left[\varepsilon_{1}\left(\bar{z}_{1}\right), \ldots, \varepsilon_{p}\left(\bar{z}_{p}\right)\right]^{T}$. Since the sign of the control gain matrix $G(x)$ is supposed known, we can propose the following fuzzy adaptive controller:

$$
\begin{aligned}
u & =D\left[\hat{\alpha}(\bar{z}, \theta)+K_{1} S+K_{0} \operatorname{Sign}(S)\right] \\
& =D\left[\theta^{T} \psi(\bar{z})+K_{1} S+K_{0} \operatorname{Sign}(S)\right],
\end{aligned}
$$

where $D=D^{-1}=\operatorname{Sign}(D)=\operatorname{diag}\left[d_{11}, d_{22}, \ldots, d_{p p}\right], K_{0}$ and $K_{1}$ are already defined in the previous section.

\section{Remark 6.}

(a) Note that the control law (63) is different from the control law (39), for the following reasons:

- The inputs of the fuzzy systems are different.

- In the control law (63), the signs of the leading principal minors of $G(x)$ are incorporated.

- In (63), the fuzzy term $\theta^{T} \psi(\bar{z})$ is used to estimate the nonlinearities $\alpha(\bar{z})$, thus this adaptive control scheme can be considered as an indirect version. In contrast, in (39), the fuzzy term $\theta^{T} \psi(\bar{z})$ is employed with an aim of estimating the ideal controller (33). Thus, the adaptive control scheme of (39) can be seen as a direct version.

- In the design of the controller (63), the matrix $G(x)$ is not assumed to be symmetric.

(b) If one chooses $\bar{z}_{i}=x$, this adaptive scheme becomes more cost-effective than the previous indirect adaptive control schemes $[3,5,8,11,13,16,19,28-30]$, since it requires $p$ fuzzy systems to implement the controller. Whereas in $[3,5,8,11,13,16,19,28-30]$, to implement the indirect control algorithm, we require $p \times p+p$ fuzzy systems: i.e. $p \times p$ fuzzy systems used to estimate the control gain matrix $G(x)$ and $p$ fuzzy systems to approximate the function (vector) $F(x)$.

Similarly to Section 4.1, after substituting (63) and (62) into (60), we can get the following dynamics:

$$
G_{1}(x) \dot{S}=-\frac{1}{2} \dot{G}_{1} S-K_{1} S-K_{0} \operatorname{Sign}(S)-\tilde{\theta}^{T} \psi(\bar{z})+\varepsilon(\bar{z}),
$$

where $D D=D D^{-1}=D \operatorname{sign}(D)=I_{p}$ and $\tilde{\theta}=\theta-\theta^{*}$. 
Multiplying $S^{T}$ to (64), we have

$$
S^{T} G_{1}(x) \dot{S}=-\frac{1}{2} S^{T} \dot{G}_{1} S-S^{T} K_{1} S-\sum_{i=1}^{p} K_{0 i}\left|S_{i}\right|-\sum_{i=1}^{p} \tilde{\theta}_{i}^{T} \psi_{i}(\bar{z}) S_{i}+S^{T} \varepsilon(\bar{z}) .
$$

To update the fuzzy parameters, we keep the adaptation PI law (42).

Theorem 3. Consider system (2). Suppose that Assumption 2 is satisfied. Then, the control law defined by (63) with the adaptation law given by (42) guarantee the following properties:

- All signals in the closed-loop system are bounded.

- The tracking errors and their derivatives decrease asymptotically to zero, i.e. $e_{i}^{(j)}(t) \rightarrow 0$ as $t \rightarrow \infty$ for $i=1, \ldots, p$ and $j=0,1, \ldots, r_{i}-1$.

Proof of Theorem 3. This proof is very similar to that of Theorem 2.

\subsection{Third fuzzy adaptive control scheme}

The two control schemes presented previously are only applied for nonlinear systems with a known sign of the control gain matrix. In this section, we propose a fuzzy adaptive controller which does not require a priori knowledge of the sign of the control gain matrix. In the control input, a Nussbaum function is incorporated, and its argument is tuned online via an appropriately designed update law. Indeed, this function can estimate the true direction of the control.

A function $N(\zeta)$ is called a Nussbaum-function, if it has the following useful properties $[10,18]$ :

(1) $\lim _{s \rightarrow+\infty} \sup \left(\frac{1}{s}\right) \int_{0}^{s} N(\zeta) d \zeta=+\infty$;

(2) $\lim _{s \rightarrow+\infty} \inf \left(\frac{1}{s}\right) \int_{0}^{s} N(\zeta) d \zeta=-\infty$.

Example. The following functions are Nussbaum functions $[10,18,33]$ :

$$
\begin{aligned}
& N_{1}(\zeta)=\zeta^{2} \cos (\zeta), \\
& N_{2}(\zeta)=\zeta \cos (\sqrt{|\zeta|}), \\
& N_{3}(\zeta)=\cos \left(\frac{\pi}{2} \zeta\right) e^{\zeta^{2}}, \\
& N_{4}(\zeta)=\ln (\zeta+1) \cos (\sqrt{\ln (\zeta+1)}) .
\end{aligned}
$$

Of course, the cosine in the above examples can be replaced by the sine. It is very easy to see that $N_{1}(\zeta), N_{2}(\zeta), N_{3}(\zeta)$ and $N_{4}(\zeta)$ are Nussbaum functions. As in [33,34], the even Nussbaum $N(\zeta)=\cos ((\pi / 2) \zeta) e^{\zeta^{2}}$ will be used throughout this paper.

In the stability analysis, we need this lemma.

Lemma 3 (Ge and Wang [10]). Let $V(\cdot)$ and $\zeta(\cdot)$ be smooth functions defined on $\left[0, t_{f}\right)$, with $V(t) \geq 0, \forall t \in\left[0, t_{f}\right)$, and $N(\cdot)$ be an even Nussbaum function. If the following inequality holds:

$$
V(t) \leq c_{0} \pm \int_{0}^{t}(g N(\zeta) \pm 1) \dot{\zeta} d \tau, \quad \forall t \in\left[0, t_{f}\right)
$$

where $g$ is non-zero constant and $c_{0}$ represents some suitable constant, then $V(t), \zeta(t)$ and $\int_{0}^{t}(g N(\zeta) \pm 1) \dot{\zeta} d \tau$ must be bounded on $\left[0, t_{f}\right)$.

Proof of Lemma 3. To proof this lemma, see [10] (in pp. 1417-1418). 
Consider again system (2) with the following assumptions.

Assumption 3. (a) The sign $G(x)$ is unknown. But, it must be positive-definite or negative-definite.

(b) $G_{s}(x)$ and $(d / d t) G_{s}(x)$ are continuous.

(c) $\partial g_{i j}(x) / \partial y_{i}^{\left(r_{i}-1\right)}=0, \forall i=1,2, \ldots, p$, and $j=1,2, \ldots, p$.

Remark 7. It is worth mentioning that in this controller the sign of $G(x)$ (or that of the matrix $D$ ) is assumed unknown but positive or negative. This is to say that the case where the sign $G(x)$ is indefinite (i.e. the matrix $D$ has +1 and -1 on its diagonal) is not included here.

Consider the following control law which incorporates the Nussbaum function

$$
\begin{aligned}
u & =N(\zeta)\left[-\hat{\alpha}(\bar{z}, \theta)-K_{1} S-K_{0} \operatorname{Sign}(S)\right] \\
& =N(\zeta)\left[-\theta^{T} \psi(\bar{z})-K_{1} S-K_{0} \operatorname{Sign}(S)\right],
\end{aligned}
$$

where

$$
N(\zeta)=\cos \left(\frac{\pi}{2} \zeta\right) e^{\zeta^{2}}
$$

and

$$
\dot{\zeta}=\sum_{i=1}^{p}\left[\theta_{i}^{T} \psi_{i}\left(\bar{z}_{i}\right)+k_{1 i} S_{i}+k_{0 i} \operatorname{Sign}\left(S_{i}\right)\right] S_{i} .
$$

Recall that $k_{0 i} \geq 0.5 \sum_{i=1}^{p} \sigma_{i}\left\|\theta_{i}^{*}\right\|^{2}+\bar{\varepsilon}_{i}$. To update the fuzzy parameters, we will keep the adaptation PI law (42).

It is very clear from (63) and (67) that the scalar function $N(\zeta)$ replaces $\operatorname{Sign}\left(-d_{i i}\right), i=1,2, \ldots, p$. Recall that $\operatorname{Sign}\left(-d_{i i}\right)=-d_{i i}$, where $d_{i i}$ are the diagonal elements of the matrix $D$. The scalar function $N(\zeta)$ is used here in order to estimate online $\operatorname{Sign}\left(-d_{i i}\right)$.

It is worth noting that, since the sign of $G(x)$ is unknown, the control law (67) can be also replaced by the following control law:

$$
u=N(\zeta)\left[\theta^{T} \psi(\bar{z})+K_{1} S+K_{0} \operatorname{Sign}(S)\right] .
$$

In this case, the Nussbaum function $N(\zeta)$ can estimate online $\operatorname{Sign}\left(d_{i i}\right)$. Later, details concerning the choice of $u$ and $\dot{\zeta}$ will be given in remark 8 .

Similarly to Sections 4.1 and 4.2, after substituting (67) into tracking error dynamics (60), we can get the following dynamics:

$$
\begin{aligned}
G_{1}(x) \dot{S} & =-0.5 \dot{G}_{1} S-K_{1} S-K_{0} \operatorname{Sign}(S)-\tilde{\theta}^{T} \psi(\bar{z})+\varepsilon(\bar{z})+\left[\theta^{T} \psi(\bar{z})+K_{1} S+K_{0} \operatorname{Sign}(S)\right]-D u \\
& =-0.5 \dot{G}_{1} S-K_{1} S-K_{0} \operatorname{Sign}(S)-\tilde{\theta}^{T} \psi(\bar{z})+\varepsilon(\bar{z})+\left[\theta^{T} \psi(\bar{z})+K_{1} S+K_{0} \operatorname{Sign}(S)\right][1+g N(\zeta)],
\end{aligned}
$$

where $g=d_{i i}, \forall i=1,2, \ldots, p$.

Multiplying (71) by $S^{T}$, we have

$$
\begin{aligned}
S^{T} G_{1}(x) \dot{S}= & -0.5 S^{T} \dot{G}_{1} S-S^{T} K_{1} S-\sum_{i=1}^{p} K_{0 i}\left|S_{i}\right|-S^{T} \tilde{\theta}^{T} \psi(\bar{z})+\sum_{i=1}^{p} \varepsilon_{i}\left(\bar{z}_{i}\right)\left|S_{i}\right| \\
& +(1+g N(\zeta))\left(\sum_{i=1}^{p} S_{i}\left[\theta_{i}^{T} \psi_{i}\left(\bar{z}_{i}\right)+k_{1 i} S_{i}+k_{0 i} \operatorname{Sign}\left(S_{i}\right)\right]\right) \\
= & -0.5 S^{T} \dot{G}_{1} S-S^{T} K_{1} S-\sum_{i=1}^{p} K_{0 i}\left|S_{i}\right|-S^{T} \tilde{\theta}^{T} \psi(\bar{z})+\sum_{i=1}^{p} \varepsilon_{i}\left(\bar{z}_{i}\right)\left|S_{i}\right|+\dot{\zeta}+g N(\zeta) \dot{\zeta}
\end{aligned}
$$


Table 1

Choices of $u$ and $\dot{\zeta}$.

\begin{tabular}{|c|c|c|c|}
\hline & Expression of $u$ & Expression of $\dot{\zeta}$ & $N(\zeta)$ estimates \\
\hline Choice 1 & $u=N(\zeta)\left[-\theta^{T} \psi(\bar{z})-K_{1} S-K_{0} \operatorname{Sign}(S)\right]$ & $\dot{\zeta}=S^{T}\left[\theta^{T} \psi(\bar{z})+K_{1} S+K_{0} \operatorname{Sign}(S)\right]$ & $\operatorname{Sign}\left(-d_{i i}\right)$ \\
\hline Choice 2 & $u=N(\zeta)\left[-\theta^{T} \psi(\bar{z})-K_{1} S-K_{0} \operatorname{Sign}(S)\right]$ & $\dot{\zeta}=-S^{T}\left[\theta^{T} \psi(\bar{z})+K_{1} S+K_{0} \operatorname{Sign}(S)\right]$ & $\operatorname{Sign}\left(-d_{i i}\right)$ \\
\hline Choice 3 & $u=N(\zeta)\left[\theta^{T} \psi(\bar{z})+K_{1} S+K_{0} \operatorname{Sign}(S)\right]$ & $\dot{\zeta}=S^{T}\left[\theta^{T} \psi(\bar{z})+K_{1} S+K_{0} \operatorname{Sign}(S)\right]$ & $\operatorname{Sign}\left(d_{i i}\right)$ \\
\hline Choice 4 & $u=N(\zeta)\left[\theta^{T} \psi(\bar{z})+K_{1} S+K_{0} \operatorname{Sign}(S)\right]$ & $\dot{\zeta}=-S^{T}\left[\theta^{T} \psi(\bar{z})+K_{1} S+K_{0} \operatorname{Sign}(S)\right]$ & $\operatorname{Sign}\left(d_{i i}\right)$ \\
\hline
\end{tabular}

Theorem 4. Consider system (2). Then, the control law defined by (67)-(69) with the adaptation law given by (42) guarantee the following properties:

- All signals in the closed-loop system are bounded.

- The tracking errors and their derivatives decrease asymptotically to zero, i.e. $e_{i}^{(j)}(t) \rightarrow 0$ as $t \rightarrow \infty$ for $i=1, \ldots, p$ and $j=0,1, \ldots, r_{i}-1$.

Proof of Theorem 4. Let us consider the following Lyapunov function candidate:

$$
V=\frac{1}{2} S^{T} G_{1}(x) S+\frac{1}{2} \sum_{i=1}^{p} \frac{1}{\gamma_{0 i}}\left(\tilde{\theta}_{i}+\gamma_{1 i} \delta_{i}\right)^{T}\left(\tilde{\theta}_{i}+\gamma_{1 i} \delta_{i}\right)
$$

Following the same stages as in Proof of Theorems 3 and 2, the time derivative of (73) can be bounded as follows:

$$
\dot{V} \leq-\sum_{i=1}^{p} k_{1 i} S_{i}^{2}+\dot{\zeta}+g N(\zeta) \dot{\zeta} .
$$

Integrating (74) over $[0, t]$, we have

$$
\begin{aligned}
V(t) & \leq V(t)+\int_{0}^{t} \sum_{i=1}^{p} k_{1 i} S_{i}^{2} d \tau \\
& \leq V(0)+\int_{0}^{t}(\dot{\zeta}+g N(\zeta) \dot{\zeta}) d \tau .
\end{aligned}
$$

According to Lemma 3, [10,34], we have $V(t), \int_{0}^{t}(1+g N(\zeta)) \dot{\zeta} d \tau, \zeta$ is bounded in $\left[0, t_{f}\right)$. Similar to discussion in [10], we know that the above discussion is also true for $t_{f}=+\infty$ (see [10,34]). Therefore $S_{i}, \tilde{\theta}_{i}+\gamma_{1 i} \delta_{i} \in L_{\infty}$. Then, from the boundedness of $S_{i}, \tilde{\theta}_{i}+\gamma_{1 i} \delta_{i}$, and $\zeta$, we can easily conclude about the boundedness of $\theta_{i}, u$, and $\bar{z}$. From (75) and since $\int_{0}^{\infty}(1+g N(\zeta)) \dot{\zeta} d \tau$ is bounded and $V(0), V(\infty) \in L_{\infty}$, it is very easy to show that $\int_{0}^{\infty} \sum_{i=1}^{p} S_{i}^{2} d t$ exists, i.e. $S_{i} \in L_{2}$. Similar to discussion in Proof of Theorems 2 and 3 , using (71) and since $S_{i}, \tilde{\theta}_{i}, u, \theta_{i}, x \in L_{\infty}$, and $G_{s}(x)=G_{1}^{-1}(x)$ and $\dot{G}_{1}(x)$ are continuous functions, we can easily show that $\dot{S}_{i} \in L_{\infty}$.

Finally, since $S_{i} \in L_{2} \cap L_{\infty}$ and $\dot{S}_{i} \in L_{\infty}$, by using Barbalat's lemma [22] we can conclude that $S_{i}(t) \rightarrow 0$ as $t \rightarrow \infty$. Therefore, the tracking errors and their derivatives converge asymptotically to zero, i.e. $e_{i}^{(j)}(t) \rightarrow 0$ as $t \rightarrow \infty$ for $i=1 \ldots p$ and $j=0,1, \ldots, r_{i}-1$.

Remark 8. We can prove thanks to Lemma 3 that there are still other possibilities for the choice of $\dot{\zeta}$ and the control $u$. Table 1 summarizes these choices. In fact, the sign of the control input gain matrix is unknown, and thus the sign choice in the expressions of $\dot{\zeta}$ and the control law $u$ is free. Similarly to Proof of Theorem 4, and using Lemma 3, we can easily show that all possible choices in Table 1 guarantee the boundedness of all involved signals in the closed-loop system and the convergence of all tracking errors to zero.

\section{Remark 9.}

(a) To eliminate the chattering effect caused by the discontinuous control term in (39), (63) and (67), the function $\operatorname{Sign}\left(S_{i}\right)$ must be replaced by any equivalent smooth function such as: $\operatorname{Tanh}\left(k_{r i} S_{i}\right), \operatorname{Arctan}\left(k_{r i} S_{i}\right)$, or $S_{i} /\left(\varepsilon_{r i}+\left|S_{i}\right|\right)$, 
$\operatorname{Sat}\left(S_{i}\right) \ldots$ where $k_{r i}, \varepsilon_{r i}>0$, with $i=1 \ldots p$. However, the use of these continuous functions only enables us to have a convergence of the tracking errors to a neighborhood of the origin.

(b) Since the value $0.5 \sigma_{i}\left\|\theta_{i}^{*}\right\|^{2}+\bar{\varepsilon}_{i}$ is unknown, the parameters $k_{0 i}$ can be estimated online by the following relation: $\dot{k}_{0 i}=\gamma_{2 i}\left|S_{i}\right|, i=1 \ldots p$, where $\gamma_{2 i}>0$.

Remark 10. As in $[3,5,8,11,13,14,16,19,28-30]$, since the fuzzy approximation (Eqs. (36) and (62)) is only guaranteed within a compact set, the stability results proposed in this work are semi-global in the sense that, for any compact set, there exists a controller with fuzzy approximation (with sufficiently large number of rules) such that all the closed-loop signals are bounded when the initial states are within this compact set. In practical applications, the number of rules usually cannot be chosen too large due to the possible computation problem. This implies that the fuzzy system approximation capability is limited, and some constraints on $\Omega_{x}$ are necessary to guarantee such an approximation.

\section{Simulation results and comparison between the three controllers}

In this section, simulation studies are carried out to show the effectiveness of the proposed adaptive fuzzy controllers. Two control problems are considered to this end. The first one concerns a two-link rigid robot manipulator moving a horizontal plane (with a symmetric control gain matrix), while the second one concerns an academic MIMO system (having a nonsymmetric control gain matrix). Moreover, a simple comparison between the three controllers is given in the end of this section.

\subsection{Example 1}

Consider a two-link rigid robot manipulator moving a horizontal plane. The dynamic equations of this MIMO system are given by $[13,22,28]$

$$
\left(\begin{array}{l}
\ddot{q}_{1} \\
\ddot{q}_{2}
\end{array}\right)=\left(\begin{array}{ll}
M_{11} & M_{12} \\
M_{21} & M_{22}
\end{array}\right)^{-1}\left\{\left(\begin{array}{l}
u_{1} \\
u_{2}
\end{array}\right)-\left(\begin{array}{cc}
-h \dot{q}_{2} & -h\left(\dot{q}_{1}+\dot{q}_{2}\right) \\
h \dot{q}_{1} & 0
\end{array}\right)\left(\begin{array}{l}
\dot{q}_{1} \\
\dot{q}_{2}
\end{array}\right)\right\},
$$

where

$$
\begin{aligned}
& M_{11}=a_{1}+2 a_{3} \cos \left(q_{2}\right)+2 a_{4} \sin \left(q_{2}\right), \\
& M_{22}=a_{2}, \\
& M_{21}=M_{12}=a_{2}+a_{3} \cos \left(q_{2}\right)+a_{4} \sin \left(q_{2}\right), \\
& h=a_{3} \sin \left(q_{2}\right)-a_{4} \cos \left(q_{2}\right),
\end{aligned}
$$

with

$$
\begin{aligned}
& a_{1}=I_{1}+m_{1} l_{c 1}^{2}+I_{e}+m_{e} l_{c e}^{2}+m_{e} l_{1}^{2}, \\
& a_{2}=I_{e}+m_{e} l_{c e}^{2}, \\
& a_{3}=m_{e} l_{1} l_{c e} \cos \left(\delta_{e}\right), \\
& a_{4}=m_{e} l_{1} l_{c e} \sin \left(\delta_{e}\right) .
\end{aligned}
$$

In the simulation, the following parameter values are used:

$$
m_{1}=1, \quad m_{e}=2, \quad l_{1}=1, \quad l_{c 1}=0.5, \quad l_{c e}=0.6, \quad I_{1}=0.12, \quad I_{e}=0.25, \quad \delta_{e}=30^{\circ} .
$$

Let $y=\left[y_{1}, y_{2}\right]^{T}=\left[q_{1}, q_{2}\right]^{T}, u=\left[u_{1}, u_{2}\right]^{T}, x=\left[q_{1}, \dot{q}_{1}, q_{2}, \dot{q}_{2}\right]^{T}$.

Then, the robot system (76) can be expressed as follows:

$$
\ddot{y}=F(x)+G(x) u,
$$


a

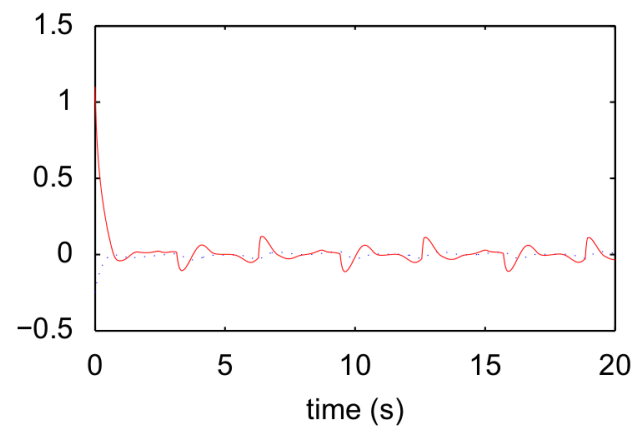

C

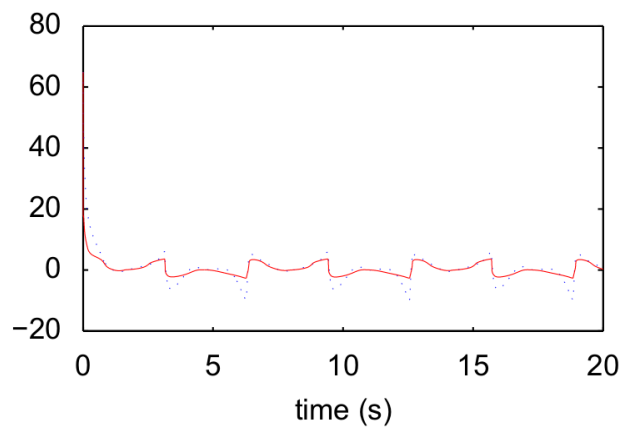

b

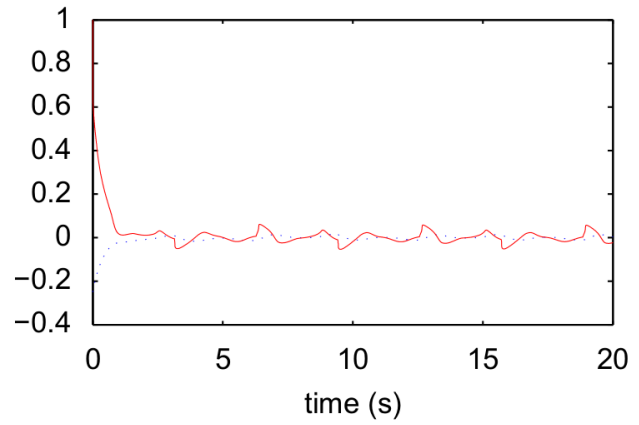

d

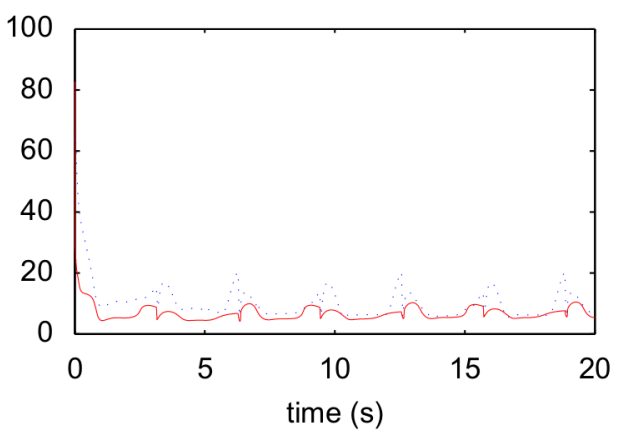

Fig. 2. Simulation results for the controller 1 (Example 1). (a) Tracking errors of link 1: $e_{1}$ (dotted line) and $\dot{e}_{1}$ (solid line). (b) Tracking errors of link 2: $e_{2}$ (dotted line) and $\dot{e}_{1}$ (solid line). (c) Control input signals: $u_{1}$ (dotted line) and $u_{2}$ (solid line). (d) Norm of fuzzy parameters: $\left\|\theta_{1}\right\|($ dotted line), $\left\|\theta_{2}\right\|$ (solid line).

where

$$
\begin{aligned}
& F(x)=\left(\begin{array}{l}
f_{1}(x) \\
f_{2}(x)
\end{array}\right)=-M^{-1}\left(\begin{array}{cc}
-h \dot{q}_{2} & -h\left(\dot{q}_{1}+\dot{q}_{2}\right) \\
h \dot{q}_{1} & 0
\end{array}\right)\left(\begin{array}{c}
\dot{q}_{1} \\
\dot{q}_{2}
\end{array}\right), \\
& G(x)=\left(\begin{array}{ll}
g_{11}(x) & g_{12}(x) \\
g_{21}(x) & g_{22}(x)
\end{array}\right)=M^{-1}=\left(\begin{array}{ll}
M_{11} & M_{12} \\
M_{21} & M_{22}
\end{array}\right)^{-1} .
\end{aligned}
$$

The control objective is to force the system outputs $q_{1}$ and $q_{2}$ to track the sinusoidal desired trajectories $y_{d 1}=\sin (t)$ and $y_{d 2}=\sin (t)$, respectively. The external disturbances are added to the system (76), they are assumed to be square waves having an amplitude \pm 1 with a period of $2 \pi(s)$.

Within all simulations, the robot dynamics are assumed completely unknown, i.e. the proposed controller does not require the knowledge of the system's model unlike in conventional model-based adaptive controllers (e.g. adaptive controllers in $[6,35])$. In fact, the dynamic model $(76)$ is only required for simulation purposes.

In all simulations, the initial conditions are selected as: $x(0)=\left[\begin{array}{llll}0.25 & 0 & 0.25 & 0\end{array}\right], \theta_{1}(0)=\underline{0}$ and $\theta_{2}(0)=\underline{0}$.

(a) Results of the first controller: It is assumed here that the sign of $G(x)$ is known. Notice that we can easily show that $G(x)$ satisfies Assumption 1.

The fuzzy systems $\theta_{2}^{T} \psi_{2}(\bar{x})$ and $\theta_{1}^{T} \psi_{1}(\bar{x})$ have $q_{1}, \dot{q}_{1}, q_{2}, \dot{q}_{2}$ as inputs. For each variable of inputs of the fuzzy systems, we define three triangular membership functions uniformly distributed on the interval $[-2,2]$.

The design parameters used in this simulation are chosen as follows: $\gamma_{01}=\gamma_{02}=500, \gamma_{11}=\gamma_{12}=500, \sigma_{1}=\sigma_{2}=$ $0,005, \lambda_{1}=\lambda_{2}=2, k_{1 i}=k_{0 i}=0.2$. Note that $k_{1 i}$ and $k_{0 i}$ are selected small in order to see the fuzzy contribution in the control law (39).

Fig. 2 shows the simulation results of the adaptive fuzzy controller (39). These results show good tracking performances with all signals in the closed-loop being bounded. Figs. 2(a) and (b) show the tracking errors of the two links. Fig. 2(c) presents the boundedness of control signals. Fig. 2(d) illustrates the norms of adaptive fuzzy parameters. 
a

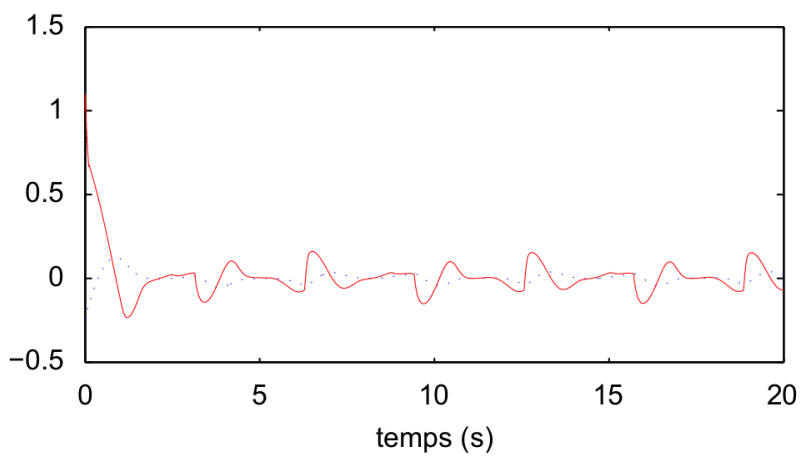

C

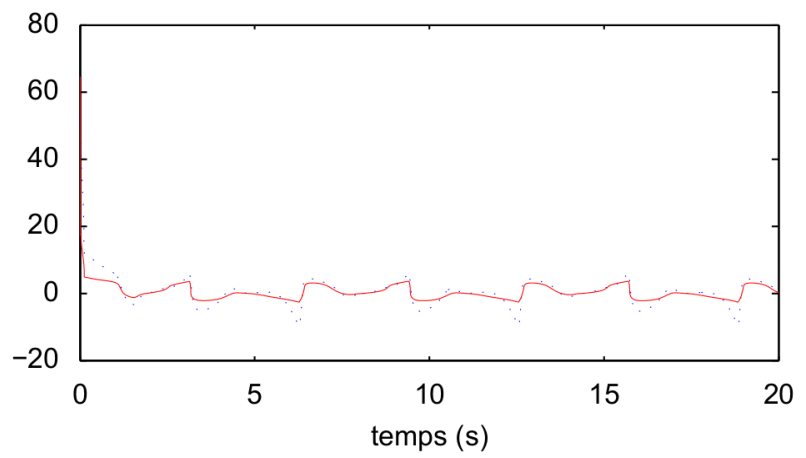

b

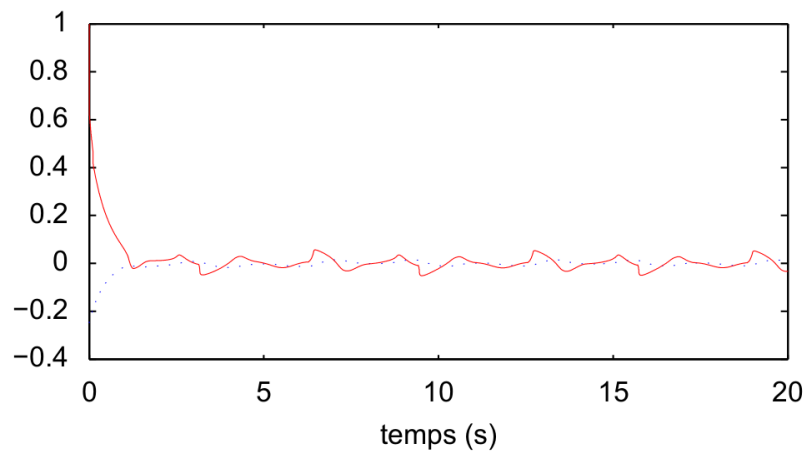

d

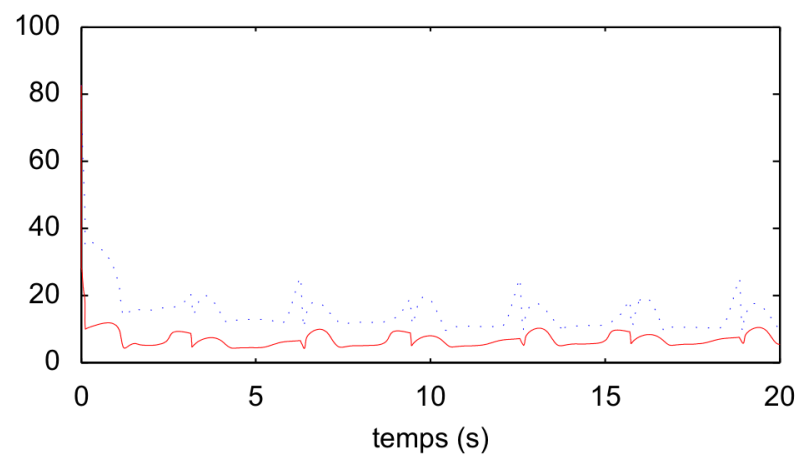

Fig. 3. Simulation results for the controller 2 (Example 1). (a) Tracking errors of link 1: $e_{1}$ (dotted line) and $\dot{e}_{1}$ (solid line). (b) Tracking errors of link 2: $e_{2}$ (dotted line) and $\dot{e}_{2}$ (solid line). (c) Control input signals: $u_{1}$ (dotted line) and $u_{2}$ (solid line). (d) Norm of fuzzy parameters: $\left\|\theta_{1}\right\|($ dotted line), $\left\|\theta_{2}\right\|$ (solid line).

(b) Results of the second controller: In this controller, the signs of diagonal elements of $D$ are assumed known. Since $G(x)$ is positive-definite, in this example we have $D=I_{p}$.

The input vectors of the fuzzy systems $\theta_{2}^{T} \psi_{2}\left(\bar{z}_{2}\right)$ and $\theta_{1}^{T} \psi_{1}\left(\bar{z}_{1}\right)$ are $\bar{z}_{2}=\left[q_{1}, \dot{q}_{1}, q_{2}, \dot{q}_{2}\right]^{T}$ and $\bar{z}_{1}=\left[q_{1}, \dot{q}_{1}, q_{2}, \dot{q}_{2}, u_{2}\right]^{T}$, respectively. We define three triangular membership functions uniformly distributed on the interval $[-2,2]$ for $q_{1}, \dot{q}_{1}, q_{2}$, and $\dot{q}_{2}$, and $[-25,25]$ for $u_{2}$. The design parameters used in this simulation are chosen as follows: $\gamma_{01}=\gamma_{02}=500$, $\gamma_{11}=\gamma_{12}=500, \sigma_{1}=\sigma_{2}=0,005, \lambda_{1}=\lambda_{2}=2, k_{1 i}=k_{0 i}=0.2$.

Fig. 3 shows the simulation results of the adaptive fuzzy controller (63). These results show also good tracking performances with all signals in the closed-loop being bounded.

(c) Results of the third controller: Note that in this controller the sign of the matrix $G(x)$ is assumed unknown. The design parameters are chosen as follows: $\gamma_{01}=\gamma_{02}=200, \gamma_{11}=\gamma_{12}=200, \sigma_{1}=\sigma_{2}=0.1, \lambda_{1}=\lambda_{2}=2$, and $k_{1 i}=k_{0 i}=0.1$. The fuzzy system $\theta_{2}^{T} \psi_{2}\left(\bar{z}_{2}\right)$ has $q_{1}, \dot{q}_{1}, q_{2}, \dot{q}_{2}$ as inputs, while $\theta_{1}^{T} \psi_{1}\left(\bar{z}_{1}\right)$ has $q_{1}, \dot{q}_{1}, q_{2}, \dot{q}_{2}, u_{2}$ as inputs. For each variable of the inputs of the fuzzy systems, we define three triangular membership functions uniformly distributed on the interval $[-2,2]$ for $q_{1}, \dot{q}_{1}, q_{2}$, and $\dot{q}_{2}$, and $[-25,25]$ for $u_{2}$.

Fig. 4 shows the simulation results obtained by using the controller (67) incorporating a Nussbaum function. From Figs. 4(a) and (b), we can see that the tracking errors are bounded and converge to zero. Fig. 4(c) illustrates that the control signals are bounded. The boundedness of the fuzzy parameters is illustrated in Fig. 4(d). The variation of the Nussbaum gain $N(\zeta)$ and that of $\zeta$ are shown in Figs. 4(e) and (f), respectively. From these figures, we can see that all signals are also bounded.

\subsection{Example 2}

Note that in the previous example the control gain matrix $G(x)$ is symmetric. In this example, we consider an academic MIMO nonlinear system of which the control gain matrix is not symmetric. The dynamic equations of this 
a

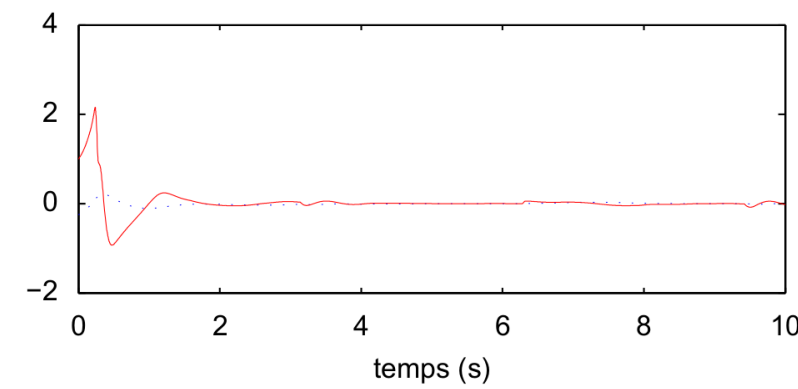

C

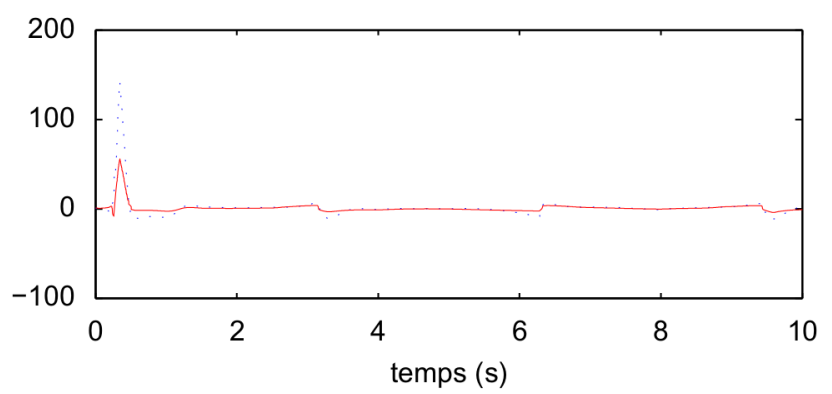

e

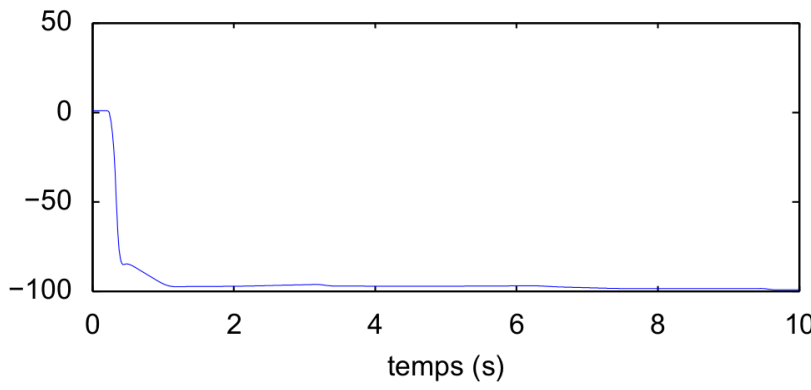

b

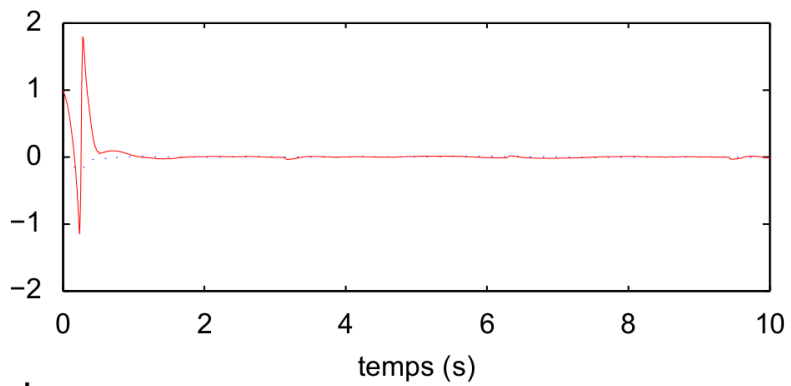

d

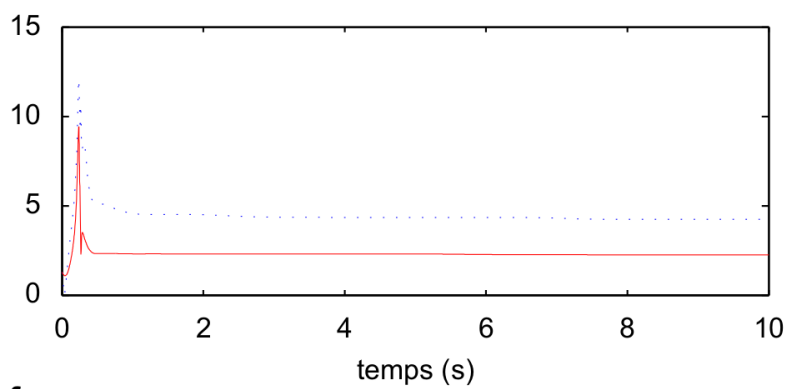

f

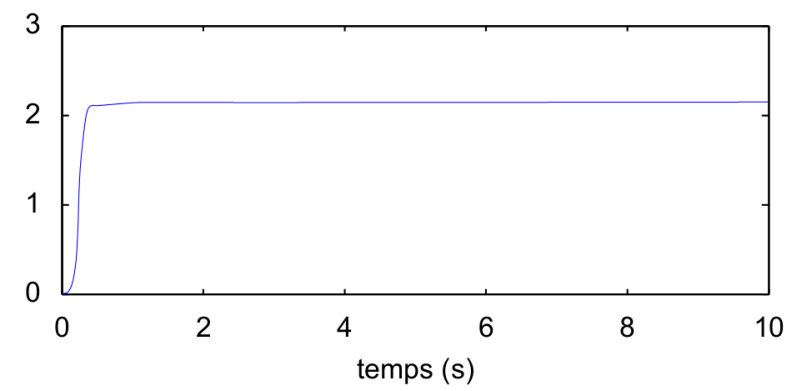

Fig. 4. Simulation results with the controller 3 (Example 1). (a) Tracking errors of link 1: $e_{1}$ (dotted line) and $\dot{e}_{1}$ (solid line). (b) Tracking errors of link 2: $e_{2}$ (dotted line) and $\dot{e}_{2}$ (solid line). (c) Control input signals: $u_{1}$ (dotted line) and $u_{2}$ (solid line). (d) Norm of fuzzy parameters: $\left\|\theta_{1}\right\|($ dotted line), $\left\|\theta_{2}\right\|$ (solid line). (e) Evolution of $N(\zeta)$. (f) Evolution of $\zeta$.

MIMO system are given by

$$
\left\{\begin{array}{l}
\dot{x}_{11}=x_{12}, \\
\dot{x}_{12}=x_{21}-0.3 \sin \left(x_{11} x_{12}\right)+x_{12}^{2}+\left(3+\cos \left(x_{11}\right)\right) u_{1}+\left(1+x_{21}^{2}\right) u_{2}+d_{1}(t), \\
\dot{x}_{21}=x_{22} \\
\dot{x}_{22}=x_{22}^{2}+x_{11}+x_{12}^{2}-u_{1}+\left(1+x_{21}^{2}\right) u_{2}+d_{2}(t), \\
y_{1}=x_{11}, \quad y_{2}=x_{2} .
\end{array}\right.
$$

Let $y=\left[y_{1}, y_{2}\right]^{T}, u=\left[u_{1}, u_{2}\right]^{T}, x=\left[x_{11}, x_{12}, x_{21}, x_{22}\right]^{T}$, and $d(t)=\left[d_{1}(t), d_{2}(t)\right]^{T}$. Then, the academic system (Eq. (78)) can be expressed as follows:

$$
\ddot{y}=F(x)+G(x) u+d(t),
$$


a

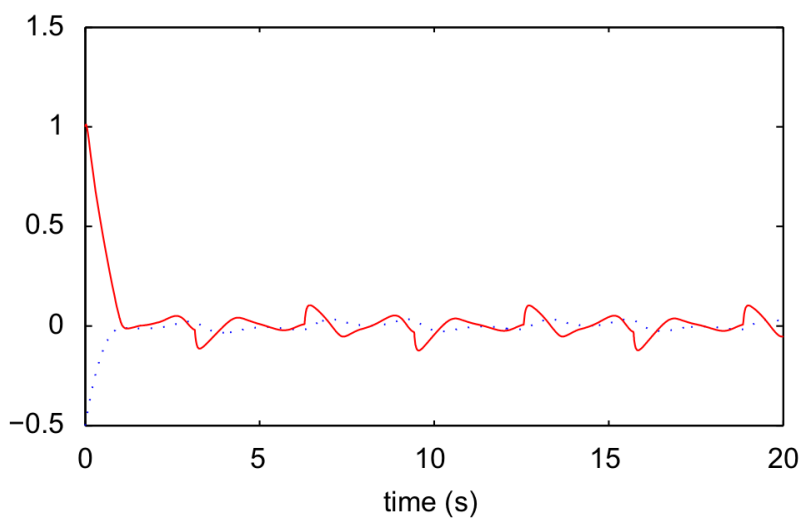

C

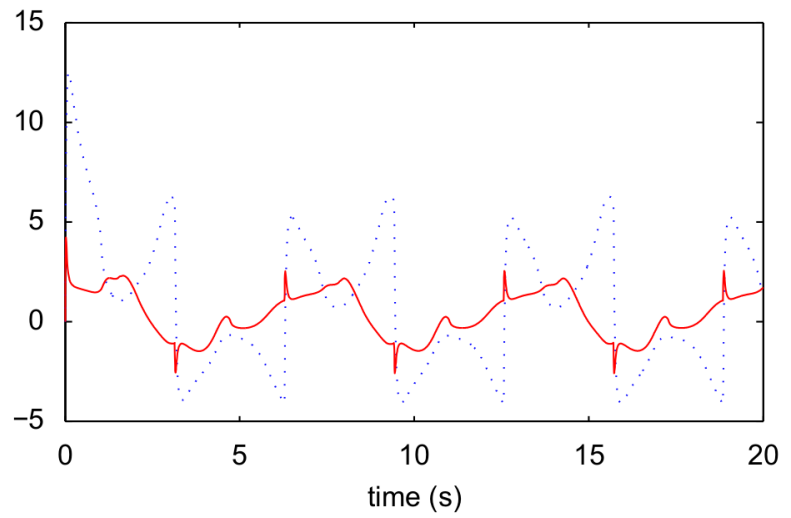

b

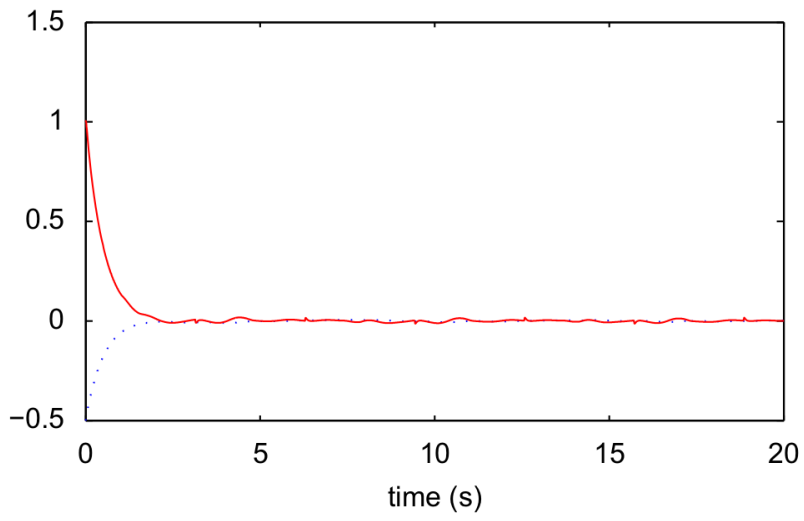

d

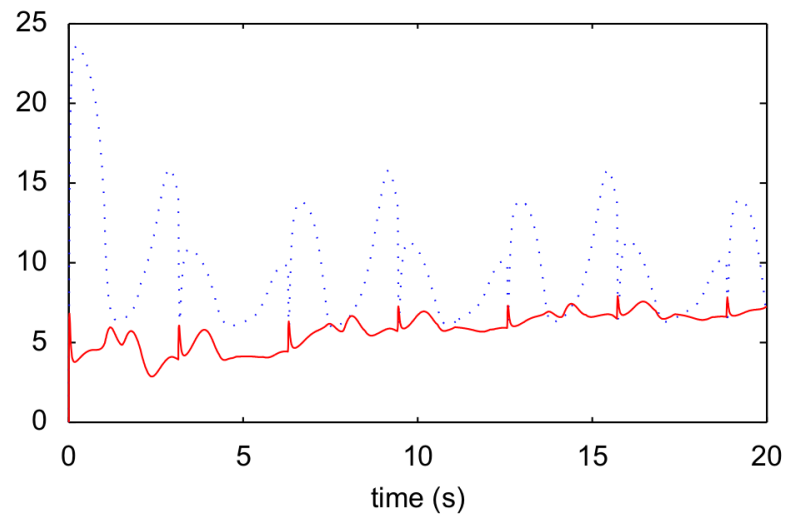

Fig. 5. Simulation results for the controller 1 (Example 2). (a) Tracking errors: $e_{1}$ (dotted line) and $\dot{e}_{1}$ (solid line). (b) Tracking errors: $e_{2}$ (dotted line) and $\dot{e}_{2}$ (solid line). (c) Control input signals: $u_{1}$ (dotted line) and $u_{2}$ (solid line). (d) Norm of fuzzy parameters: $\left\|\theta_{1}\right\|$ (dotted line), $\left\|\theta_{2}\right\|($ solid line).

where

$$
F(x)=\left(\begin{array}{c}
x_{21}-0.3 \sin \left(x_{11} x_{12}\right)+x_{12}^{2} \\
x_{22}^{2}+x_{11}+x_{12}^{2}
\end{array}\right), \quad G(x)=\left(\begin{array}{cc}
\left(3+\cos \left(x_{11}\right)\right) & \left(1+x_{21}^{2}\right) \\
-1 & \left(1+x_{21}^{2}\right)
\end{array}\right),
$$

The control objective is to force the system outputs $y_{1}$ and $y_{2}$ to track the sinusoidal desired trajectories $y_{d 1}=\sin (t)$ and $y_{d 2}=\sin (t)$, respectively. The external disturbances $d_{1}(t)$ and $d_{2}(t)$ are assumed to be square waves having an amplitude \pm 1 with a period of $2 \pi(s)$.

In each controller, the structure of the fuzzy systems (i.e. the inputs of the fuzzy systems, the universes of discourse, the number and the type of the membership functions) is selected similar to that of Example 1. In all simulations, the initial conditions are selected as: $x(0)=\left[\begin{array}{llll}0.5 & 0 & 0.5 & 0\end{array}\right], \theta_{1}(0)=\underline{0}$ and $\theta_{2}(0)=\underline{0}$.

(a) Results of the first controller: The design parameters used in this simulation are chosen as follows: $\gamma_{01}=\gamma_{02}=$ $200, \gamma_{11}=\gamma_{12}=200, \sigma_{1}=\sigma_{2}=0,02, \lambda_{1}=\lambda_{2}=2, k_{1 i}=k_{0 i}=0.2$.

Fig. 5 shows the simulation results obtained using the adaptive controller (39). It can be seen that fairly good tracking performance is obtained.

(b) Results of the second controller: The values of the design parameters are given by: $\gamma_{01}=\gamma_{02}=200, \gamma_{11}=$ $\gamma_{12}=200, \sigma_{1}=\sigma_{2}=0,02, \lambda_{1}=\lambda_{2}=2, k_{1 i}=k_{0 i}=0.2$.

The simulation results using the adaptive controller (63) are shown in Fig. 6. From these results, it is shown that the performance of this controller is also satisfactory. 
a

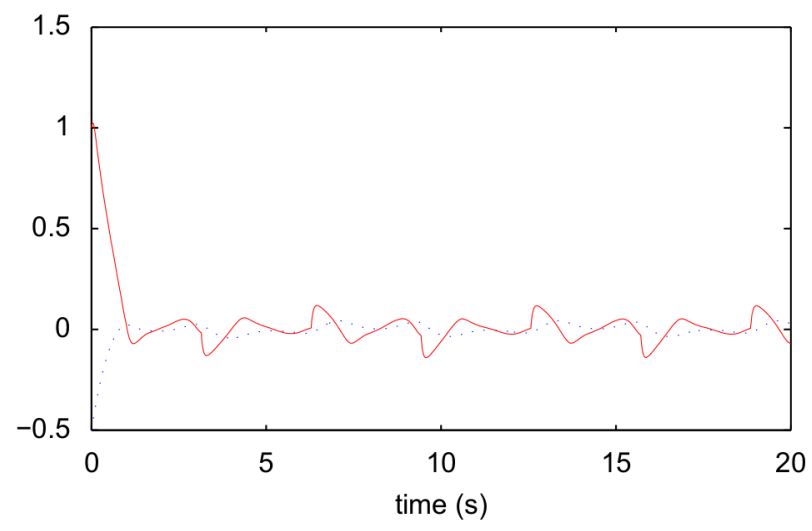

C

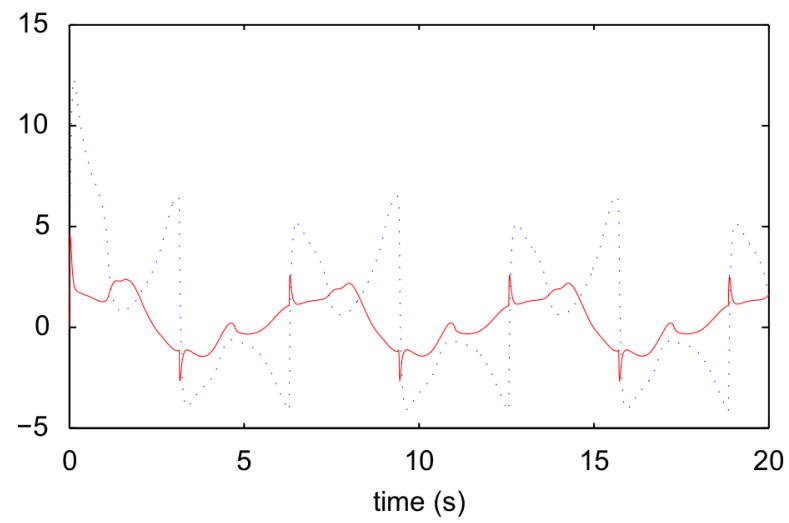

b

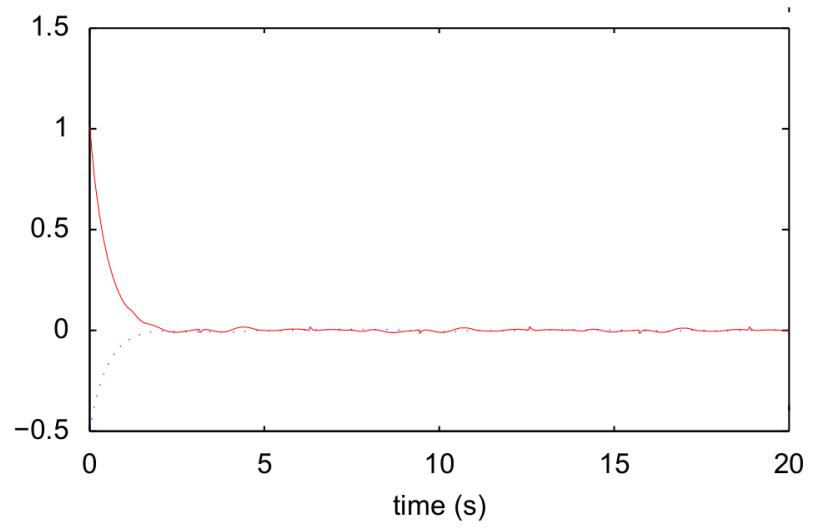

d

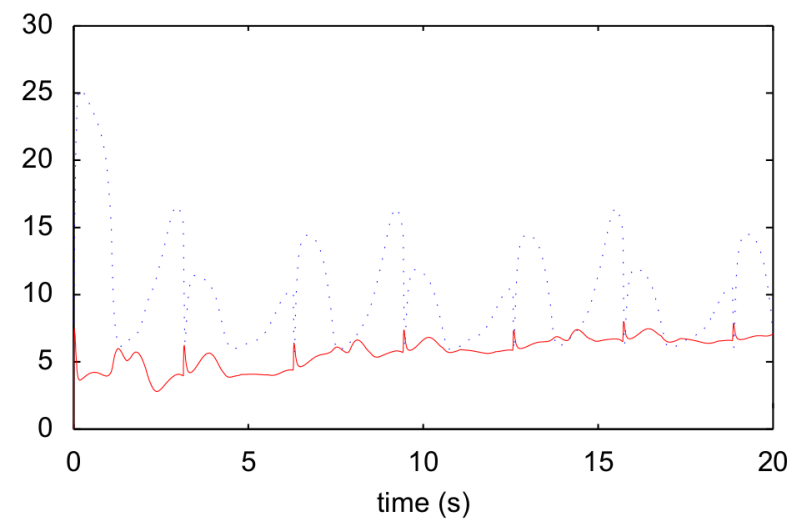

Fig. 6. Simulation results for the controller 2 (Example 2). (a) Tracking errors: $e_{1}$ (dotted line) and $\dot{e}_{1}$ (solid line). (b) Tracking errors: $e_{2}$ (dotted line) and $\dot{e}_{2}$ (solid line). (c) Control input signals: $u_{1}$ (dotted line) and $u_{2}$ (solid line). (d) Norm of fuzzy parameters: $\left\|\theta_{1}\right\|$ (dotted line), $\left\|\theta_{2}\right\|$ (solid line).

(c) Results of the third controller: The values of the design parameters are given by: $\gamma_{01}=\gamma_{02}=100, \gamma_{11}=\gamma_{12}=$ $100, \sigma_{1}=\sigma_{2}=0,02, \lambda_{1}=\lambda_{2}=2, k_{1 i}=k_{0 i}=0.2$.

The simulation results using the adaptive controller (67) are shown in Fig. 7.

\subsection{Comparison between the three controllers}

In both examples, the results of simulation show clearly that the performances of controllers 1 and 2 are perfectly the same. In spite of its average transient behavior, steady-state performances of the third controller are very good. In fact, the function of Nussbaum amplified the control signal. The rest of this comparison is summarized in Table 2.

\section{Conclusion}

In this paper, fuzzy adaptive control schemes for a class of MIMO unknown nonlinear systems with known and unknown sign of the control gain matrix have been presented. To deal with the unknown control direction, the Nussbaumtype function has been used. In the designing of the second and third fuzzy adaptive controller, the decomposition property of the control gain matrix has been exploited. For updating the fuzzy parameters, an adaptation PI law has been proposed. It has been proven that the proposed control schemes can guarantee the convergence of the tracking errors to zero and the boundedness of all involved signals in the closed-loop system. Simulation results have shown the effectiveness of the proposed controllers. 
a

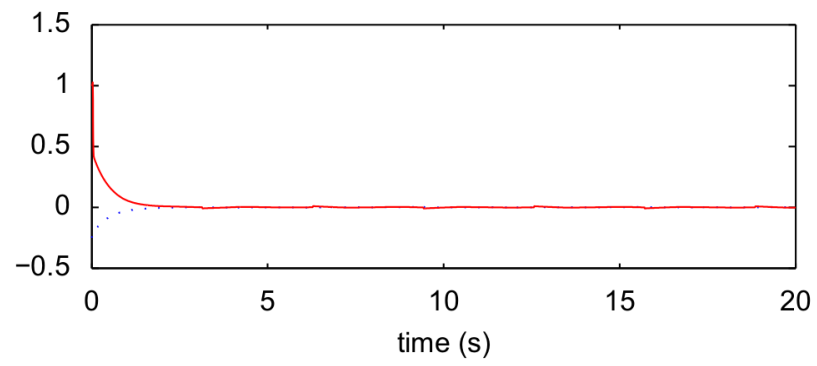

C

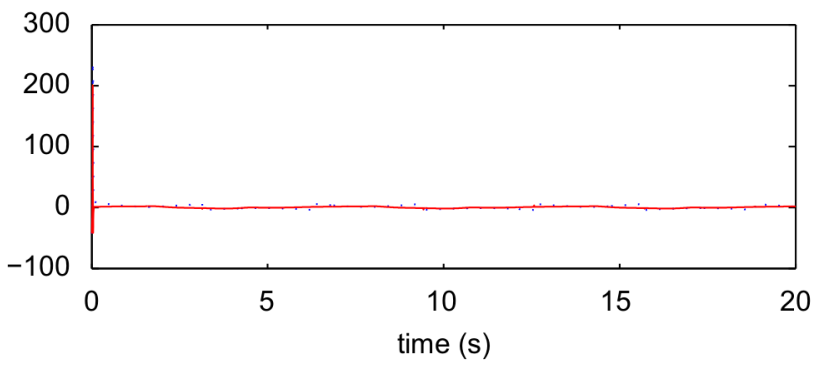

e

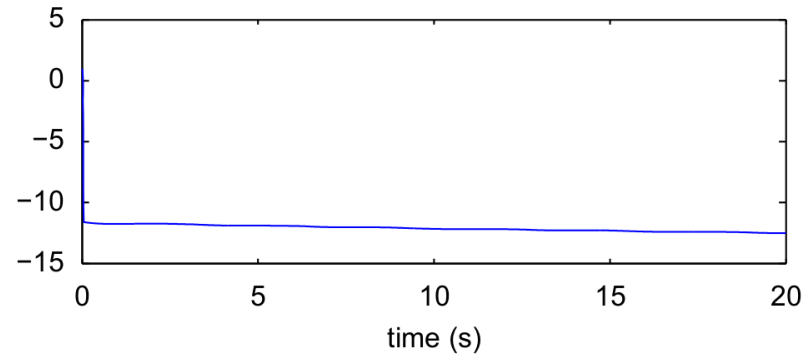

b

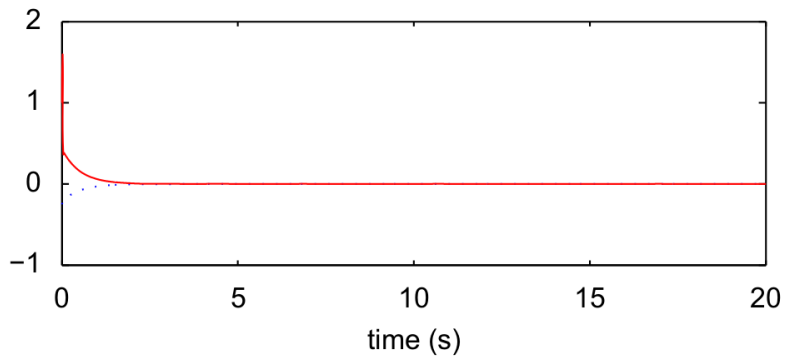

d

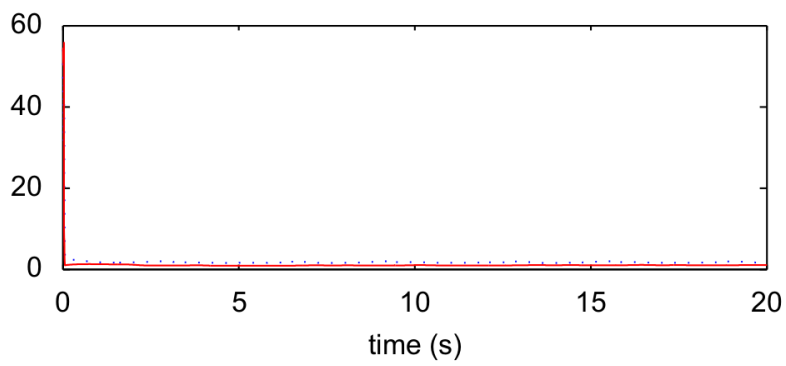

f

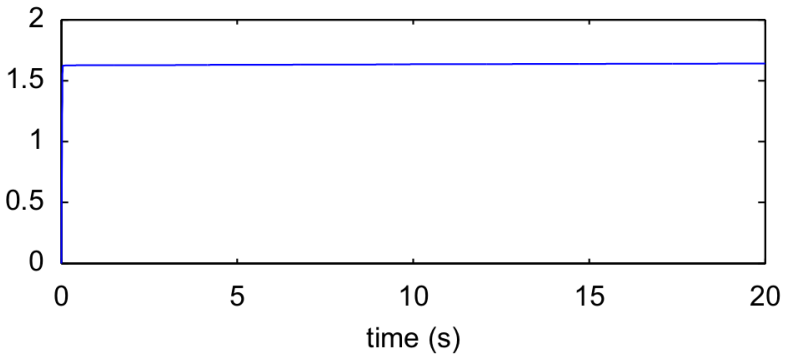

Fig. 7. Simulation results with the controller 3 (Example 2). (a) Tracking errors: $e_{1}$ (dotted line) and $\dot{e}_{1}$ (solid line). (b) Tracking errors: $e_{2}$ (dotted line) and $\dot{e}_{2}$ (solid line). (c) Control input signals: $u_{1}$ (dotted line) and $u_{2}$ (solid line). (d) Norm of fuzzy parameters: $\left\|\theta_{1}\right\|$ (dotted line), $\left\|\theta_{2}\right\|$ (solid line). (e) Evolution of $N(\zeta)$. (f) Evolution of $\zeta$.

Table 2

Comparison between the three controllers.

\begin{tabular}{|c|c|c|c|}
\hline & Controller 1 & Controller 2 & Controller 3 \\
\hline Assumption made on the sign of $G(x)$ & $\begin{array}{l}\text { Positive-definite but with } \\
\text { known sign }\end{array}$ & $\begin{array}{l}\text { Signs of its leading principal } \\
\text { minors are known }\end{array}$ & $\begin{array}{l}\text { Positive-definite or } \\
\text { negative-definite but } \\
\text { with unknown sign }\end{array}$ \\
\hline Symmetry of the $G(x)$ & Necessary & Not necessary & Not necessary \\
\hline Tracking performances in simulation results & Good & Good & Very good \\
\hline The type of the adaptive scheme & Direct & Indirect & Indirect \\
\hline
\end{tabular}

\section{References}

[1] A. Boulkroune, M. Tadjine, M. Msaad, M. Farza, A unified approach for design of indirect adaptive output-feedback fuzzy controller, Int. J. Intell. Systems Technol. Appl. 5 (1/2) (2008) 83-103.

[2] A. Boulkroune, M. Tadjine, M. Msaad, M. Farza, How to design a fuzzy adaptive control based on observers for uncertain affine nonlinear systems, Fuzzy Sets and Systems 159 (2008) 926-948. 
[3] Y.C. Chang, Robust tracking control for nonlinear MIMO systems via fuzzy approaches, Automatica 36 (2000) 1535-1545.

[4] Y.C. Chang, Adaptive fuzzy-based tracking control for nonlinear SISO systems via VSS and $H^{\infty}$ approaches, IEEE Trans. Fuzzy Systems 9 (2001) 278-292.

[5] H. Chekireb, M. Tadjine, D. Bouchaffra, Direct adaptive fuzzy control of nonlinear system class with applications, Control Intell. Systems 31 (2) (2003) $1-11$.

[6] J. Chen, A. Behal, D.M. Dawson, Adaptive output feedback control for a class of MIMO nonlinear systems, in: Proc. American Control Conf., Minneapolis, MN, 2006, pp. 5300-5305.

[7] R.R. Costa, L. Hsu, A.K. Imai, P. Kokotovic, Lyapunov-based adaptive control of MIMO systems, Automatica 39 (7) (2003) 1251 -1257.

[8] N. Essounbouli, A. Hamzaoui, J. Zaytoon, An improved robust adaptive fuzzy controller for MIMO systems, Control Intell. Systems 34 (1) (2006) 12-21.

[9] S.S. Ge, C.C. Hang, T.H. Lee, T. Zhang, Stable Adaptive Neural Network Control, Kluwer Academic Publisher, Boston, 2001.

[10] S.S. Ge, J. Wang, Robust adaptive neural control for a class of perturbed strict feedback nonlinear systems, IEEE Trans. Neural Networks 13 (6) (2002) 1409-1419.

[11] N. Golea, A. Golea, K. Benmahammed, Stable indirect fuzzy adaptive control, Fuzzy Sets and Systems 137 (2003) $353-366$.

[12] L. Hsu, R.R. Costa, F. Lizarralde, Lyapunov/passivity-based adaptive control of relative degree two MIMO systems with an application to visual servoing, IEEE Trans. Automat. Control 52 (2) (2007) 364-371.

[13] S. Labiod, M.S. Boucherit, T.M. Guerra, Adaptive fuzzy control of a class of MIMO nonlinear systems, Fuzzy Sets and Systems 151 (2005) 59-77.

[14] S. Labiod, T.M. Guerra, Direct adaptive fuzzy control for a class of MIMO nonlinear systems, Int. J. Systems Sci. 38 (8) (2007) $665-675$.

[15] H. Lee, M. Tomizuka, Robust adaptive control using a universal approximator for SISO nonlinear systems, IEEE Trans. Fuzzy Systems 8 (1) (2000) $95-106$.

[16] H.X. Li, S.C. Tong, A hybrid adaptive fuzzy control for a class of nonlinear MIMO systems, IEEE Trans. Fuzzy Systems 11 (1) (2003) 24-34.

[17] K.S. Narendra, M.A. Annaswamy, A new adaptive law for robust adaptive control without persistent excitation, IEEE Trans. Automat. Control 32 (1987) 134-145.

[18] R.D. Nussbaum, Some remarks on the conjecture in parameter adaptive control, Systems Control Lett. 3 (5) (1983) $243-246$.

[19] R. Ordonez, K.M. Passino, Stable multi-input multi-output adaptive fuzzy/neural control, IEEE Trans. Fuzzy Systems 7 (3) (1999) $345-353$.

[20] S. Perlis, Theory of Matrices, Dover Publications, New York, 1991.

[21] R.M. Sanner, J.E. Slotine, Gaussian networks for direct adaptive control, IEEE Trans. Neural Networks 3 (6) (1992) $837-863$.

[22] J.E. Slotine, W. Li, Applied Nonlinear Control, Prentice-Hall, Englewood Cliffs, NJ, 1991.

[23] J.T. Spooner, K.M. Passino, Stable adaptive control using fuzzy systems and neural networks, IEEE Trans. Fuzzy Systems 4 (1996) $339-359$.

[24] G. Strang, Linear Algebra and its Applications, Academic Press Inc., New York, 1980.

[25] C.Y. Su, Y. Stepanenko, Adaptive control of a class of nonlinear systems with fuzzy logic, IEEE Trans. Fuzzy Systems 2 (1994) $285-294$.

[26] Y. Tang, N. Zhang, Y. Li, Stable fuzzy adaptive control for a class of nonlinear systems, Fuzzy Sets and Systems 104 (1999) $279-288$.

[27] S.C. Tong, Q. Li, T. Chai, Fuzzy adaptive control of a class of nonlinear systems, Fuzzy Sets and Systems 101 (1999) 31-39.

[28] S.C. Tong, J. Tang, T. Wang, Fuzzy adaptive control of multivariable nonlinear systems, Fuzzy Sets and Systems 111 (2) (2000) $153-167$.

[29] S.C. Tong, H.X. Li, Fuzzy adaptive sliding model control for MIMO nonlinear systems, IEEE Trans. Fuzzy Systems 11 (3) (2003) 354-360.

[30] S.C. Tong, B. Chen, Y. Wang, Fuzzy adaptive output feedback control for MIMO nonlinear systems, Fuzzy Sets and Systems 156 (2) (2005) 285-299.

[31] L.X. Wang, Adaptive Fuzzy Systems and Control: Design and Stability Analysis, Prentice-Hall, Englewood Cliffs, NJ, 1994.

[32] X.D. Ye, Adaptive nonlinear output-feedback control with unknown high-frequency gain sign, IEEE Trans. Automat. Control 46 (1) (2001) 112-115.

[33] T.P. Zhang, S.S. Ge, Adaptive neural control of MIMO nonlinear state time-varying delay systems with unknown dead-zones and gain signs, Automatica 43 (6) (2007) 1021-1033.

[34] T.P. Zhang, Y. Yi, Adaptive fuzzy control for a class of MIMO nonlinear systems with unknown dead-zones, Acta Automat. Sinica 33 (1) (2007) 96-99.

[35] X.T. Zhang, D.M. Dawson, M.S. De Queiroz, B. Xian, Adaptive control for a class of MIMO nonlinear systems with non-symmetric input matrix, in: Proc. IEEE International Conf. on Control Applications, Taipei, Taiwan, 2004, pp. 1324-1329. 